\title{
Response attenuation in a large-scale structure subjected to blast excitation utilizing a system of essentially nonlinear vibration absorbers
}

\author{
Nicholas E. Wierschem, Ph.D. ${ }^{1}$, Sean A. Hubbard, Ph.D. ${ }^{2}$, Jie Luo ${ }^{3}$, Larry A. Fahnestock, \\ Ph.D., P.E. ${ }^{4}$, Billie F. Spencer, Jr., Ph.D., P.E. ${ }^{5}$, D. Michael McFarland, Ph.D. ${ }^{6}$, D. Dane Quinn, \\ Ph.D. ${ }^{7}$, Alexander F. Vakakis, Ph.D. ${ }^{8}$, and Lawrence A. Bergman, Ph.D. ${ }^{9}$
}

\begin{abstract}
Limiting peak stresses and strains in a structure subjected to high-energy, short-duration transient loadings, such as blasts, is a challenging problem, largely due to the well-known insensitivity of the first few cycles of the structural response to damping. Linear isolation, while a potential solution, requires a very low fundamental natural frequency to be effective, resulting in large nearly-rigid body displacement of the structure, while linear vibration absorbers have little or no effect on the early-time response where relative motions, and thus stresses and strains, are at their highest levels. The problem has become increasingly important in recent years with the expectation of blast-resistance as a design requirement in new construction. In this paper, the problem is examined experimentally and computationally in the context of offset-blast loading applied to a custom-built nine story steel frame structure. A fully-passive response mitigation system consisting of six lightweight, essentially nonlinear vibration absorbers (termed nonlinear energy sinks - NESs) is optimized and deployed on the upper two floors of this structure. Two
\end{abstract}


NESs have vibro-impact nonlinearities and the other four possess smooth but essentially nonlinear stiffnesses. Results of the computational and experimental study demonstrate the efficacy of the proposed passive nonlinear mitigation system to rapidly and efficiently attenuate the global structural response, even at early time (i.e., starting at the first response cycle), thus minimizing the peak demand on the structure. This is achieved by nonlinear redistribution of the blast energy within the modal space through low-to-high energy scattering due to the action of the NESs. The experimental results validate the theoretical predictions.

Key words: Blast mitigation, nonlinear energy sink, modal energy redistribution, passive control

\section{Introduction}

Over the past several decades, intentional and accidental explosions, and other blast events have critically damaged building structures [1]. This vulnerability has motivated a significant body of research addressing the blast resistance of structures, with many recent efforts focused on local effects of the blast, progressive collapse, and resistance of nonstructural elements. Despite this recent focus, structures remain vulnerable to failure mechanisms related to their global response [2]. Furthermore, as structures subjected to blast become more impervious to local component-level damage such that structure-level mechanisms govern, controlling the global response to offset-blast loads becomes increasingly important.

Over the past fifteen years the ability of a class of strongly nonlinear vibration absorbers or nonlinear energy sinks (NESs) to rapidly attenuate the global response of structures subjected to impulsive-type loads has been studied analytically and computationally, and demonstrated experimentally [3-11]. NESs are a class of vibrating attachments with the capacity to passively absorb and locally dissipate vibration energy from arbitrary modes of a base structure to which it's attached in a broadband or narrowband fashion; however, unlike traditional linear and weakly nonlinear absorbers, NESs employ strongly nonlinear stiffness elements that produce essentially nonlinear (that is, non-linearizable) restoring forces. Thus, the response of the NES is not dominated by a single preferential natural frequency, and so the NES has the ability engage in transient resonance capture [12] with multiple modes of the base structure, resulting in multifrequency Targeted Energy Transfer (TET) [13]. The strong nonlinearity also affects the global response of the system, facilitating the transfer of energy from lower modes of vibration to higher ones where it is more effectively dissipated by the inherent damping of the base structure itself. Much of this early work focused on small-scale structures with a single NES subjected to impulsive loading and served to effectively demonstrate the capability of TET to quickly mitigate the effects of high energy, short duration loads on structures.

In this paper, offset-blast loading applied to a large-scale steel frame structure equipped with a system of NESs is examined; this represents the first time a large-scale structure with an NES 
or system of NESs has been experimentally tested under actual blast loading, and in that context this work differs from previous works that consider passive nonlinear mitigation of this structure either computationally or by means of experimental shaker tests. The protective system designed for this study consists of two types of NESs. The first, referred to as Type I, employs a smooth restoring force that is ideally purely cubic $[14,15]$. The second, referred to as Type VI or SSVI (single-sided vibro-impact) [7], utilizes a very weak linear restoring force coupled to a mass undergoing one-sided vibro-impacts [16]. The paper begins with a description of the base structure and protective system, including a discussion of the NES modeling and parameter optimization. Then the blast loading is discussed as well as how it is achieved in the performed experiments. The results of multiple blast tests are then presented in order to evaluate the efficacy of the protective system of NESs over a range of load intensities. Experimental results are compared with those of computational simulations to demonstrate predictive capability, and results from additional computational simulations are also presented.

\section{The Structural System}

The structural system employed in this work consists of a large-scale nine-story steel frame $[3,17]$, referred to hereafter as the base structure, and a system of six NESs. The NESs are built directly and nonparasitically into the two highest floors of the base structure; that is, the mass of material removed from the floors to accommodate the NESs approximately offsets the mass of the NESs.

\subsection{Base structure}

The base structure is shown in Figure 1. This structure, which was designed and built specifically for this project, is $5.13 \mathrm{~m}$ tall and has a mass approximately equal to $11,000 \mathrm{~kg}$. To the authors' knowledge, this makes the structure the largest existing test bed for NES technology, substantially larger in both height and mass than the test bed discussed in [11]. It consists of nine $2.74 \mathrm{~m}$ by $1.22 \mathrm{~m}$ steel floor plates, and one $2.90 \mathrm{~m}$ by $1.22 \mathrm{~m}$ steel base plate. The bottom seven floors are solid $3.81 \mathrm{~cm}$ thick plates, while the base plate and the top two floors are 4.44 $\mathrm{cm}$ thick. Additionally, the two top floor plates are cut out to accommodate the NESs. At the test site, the structure is connected, via the base plate, to a poured concrete pad. The mass and dimensions of this pad are sufficient to ensure that, during the blast testing, the base of the structure is secured against movement.

An identical column layout is used for each floor of the base structure. As shown in Figure 1, this layout consists of eight columns arranged such that there is one bay in the short direction of the plates and three bays in the long direction. The columns are high-strength steel to allow the building to elastically accommodate relatively large deformations. All columns in the structure are rectangular with those on the bottom floor being $66.04 \mathrm{~cm}$ tall with a $19.05 \mathrm{~cm}$ by $1.43 \mathrm{~cm}$ cross-section. The remaining columns, on all other floors, are $50.80 \mathrm{~cm}$ tall with a $13.97 \mathrm{~cm}$ by $1.43 \mathrm{~cm}$ cross-section. Because of their aspect ratios, the bending stiffness of each 


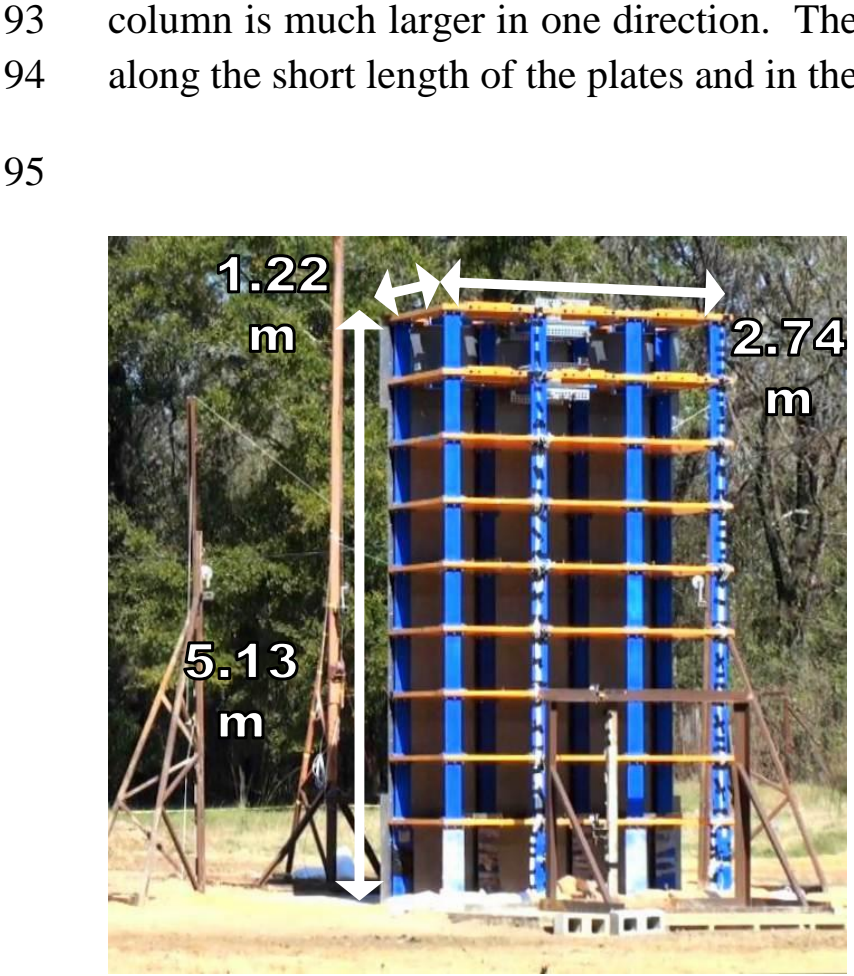

(a)

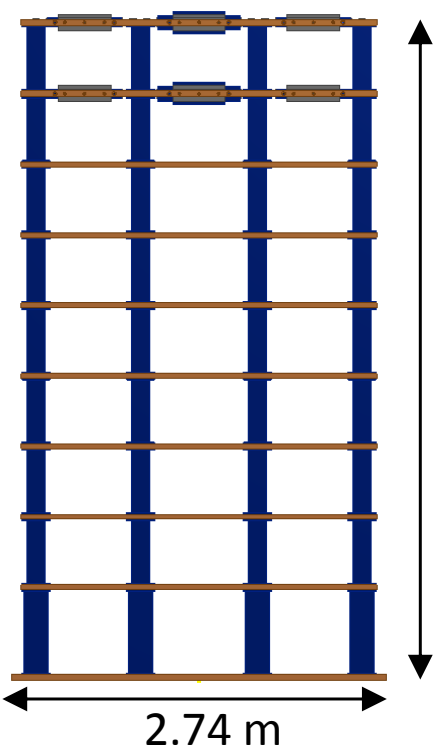

(b)

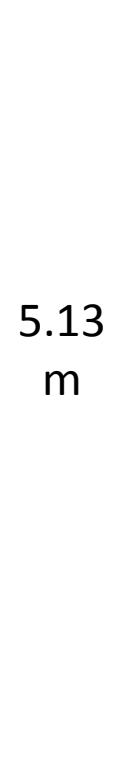

Figure 1. Base Structure with system of six NESs visible on the two top floors (a) setup at test site, (b) front view drawing with cladding removed, and (c) side view drawing with cladding removed.

Steel cladding covers one of the long sides of the structure to react the loading, which is offset from the cladded side. The cladding acts to transfer the pressure load that accumulates on it to the structure. It is composed of a series of overlapping $0.13 \mathrm{~cm}$ thick steel plates. Most of these plates are $274 \mathrm{~cm}$ by $107 \mathrm{~cm}$, which permits each cladding section to span the distance between adjacent floors. Overlapped sections of cladding at levels one through eight are connected to the structure via a row of 21 bolts driven directly into the side of the floor slab. At these levels the overlapping cladding plates are also connected to each other via two rows of bolts above and below the floor plate connection. At the top of the structure, the ninth floor, one section of cladding is connected to the floor plate with a row of bolts. Additionally, the top plate is folded over and connected vertically to the plate with bolts. At the bottom of the structure, one section of cladding is attached to the structure via a row of bolts. This bottom section of cladding is folded such that it is directly connected to the foundation via a row of anchors embedded in the concrete slab. Because of the relatively small mass of the cladding and its flexibility in the weak translational direction of the structure, its effect on the weak direction translational natural frequencies is relatively small. However, larger stiffnesses in torsion and in the strong translational direction result in significant increases in torsional and strong direction translational natural frequencies. 


\subsection{System of nonlinear energy sinks (NESs)}

Six NESs are installed in the base structure, located within cutouts built into the floor plates of the eighth and ninth floors. The installation, nearly identical for both floors, is shown in Figure 2. As shown, three NESs are built into each floor; the outer two NESs are of Type I, whereas the center NES is of Type VI (or SSVI). Conceptual models of these NESs connected to a single-degree-of-freedom linear oscillator are shown in Figure 3 . The idealized representations of both types of NESs consist of masses, springs, and dampers. The conceptual model of the Type VI NES is a mass attached to a base structure through a viscous damper and very weak linear spring; here, the displacement of the mass is limited on one side only by an impact surface connected to the base structure. The equations of motion for this NES connected to a single-degree-of-freedom linear oscillator in between impacts is

$$
\begin{gathered}
m_{b} \ddot{x}_{b}+c \dot{x}_{b}+k_{b} x_{b}-c\left(\dot{x}-\dot{x}_{b}\right)-k_{\text {lin }}\left(x-x_{b}\right)=0 \\
m \ddot{x}+c\left(\dot{x}-\dot{x}_{b}\right)+k_{\text {lin }}\left(x-x_{b}\right)=0
\end{gathered}
$$

where $m$ is the mass of the NES, $m_{b}$ is the mass of the base, $c$ is the NES's viscous damping coefficient, $c_{b}$ is the viscous damping coefficient of the base, $k_{\text {lin }}$ is the NES's linear stiffness coefficient, $k_{b}$ is the stiffness coefficient of the base, $x$ is the displacement of the NES, and $x_{b}$ is the displacement of the base. When the displacement of the NES, relative to the displacement of the base mass it is attached to, reaches the clearance value, $x_{\text {clear }}$, an impact occurs. This impact can be modeled as an inelastic collision in which momentum is conserved [7]. The resulting velocity of the NES mass and base mass after this collision is

$$
\dot{x}_{b}^{+}=\frac{\dot{x}_{b}^{-}\left(1-\varepsilon r_{c}\right)+\dot{x}^{-}\left(1+r_{c}\right)}{1+\varepsilon}, \quad \dot{x}^{+}=\frac{\dot{x}_{b}^{-}\left(1+r_{c}\right)+\dot{x}^{-}\left(\varepsilon+r_{c}\right)}{1+\varepsilon}
$$

where the superscript ${ }^{+}$denotes the state of the system just after the impact, the superscript denotes the state of the system just before the impact, $r_{c}$ is the restitution coefficient, and $\varepsilon$ is the ratio of NES mass to the base mass it is colliding with. The coefficient of restitution of the impact is dependent on the material of the masses that take part in the impact. The result of these single-sided vibro-impacts is a sharp discontinuity in the restoring force. These discontinuities give rise to broadband discrete-time events and, because of them, energy is scattered throughout the base structure, particularly to its higher modes [7]. This is the primary reason for introducing the Type VI NESs: Due to their fast reaction time (indeed vibro-impacts are initiated during the first cycle of the structural response), they are designed to rapidly and efficiently transfer blast energy from the lower high-amplitude structural modes (that typically are excited by the blast) to the higher lower-amplitude modes. Apart from an immediate structural response reduction, such nonlinear energy scattering enables the better utilization of the inherent damping capacity of the structure itself; this is due to the fact that higher structural modes are more efficient energy 
dissipators than lower ones. Moreover, the nonlinear energy scattering also "feeds" blast energy to the Type I (smooth) NESs which is locally dissipated there by their damping elements. Hence, the Type VI NESs induce, in effect, a nonlinear energy redistribution of the blast energy within the modal space of the base structure and the system of NESs, which leads to effective and rapid response reduction and passive energy dissipation. We note that, by their vibro-impact nature, the Type VI NESs are early time devices designed to operate in the initial highly energetic phase of the response (immediately after the blast), thus addressing the need to quickly counteract and mitigate the blast energy transmitted to the base structure.

As shown in Figure 3, the conceptual model of the Type I NES consists of a mass connected to the base structure through a viscous damper and a smooth approximately pure cubic spring. The equations of motion for this NES connected to a single-degree-of-freedom linear oscillator is

$$
\begin{gathered}
m_{b} \ddot{x}_{b}+c \dot{x}_{b}+k_{b} x_{b}-c\left(\dot{x}-\dot{x}_{b}\right)-k_{n l}\left(x-x_{b}\right)^{3}=0 \\
m \ddot{x}+c\left(\dot{x}-\dot{x}_{b}\right)+k_{n l}\left(x-x_{b}\right)^{3}=0
\end{gathered}
$$

161 where $m$ is the mass of the NES, $m_{b}$ is the mass of the base, $c$ is the NES's viscous damping coefficient, $c_{b}$ is the viscous damping coefficient of the base, $k_{n l}$ is the coefficient on the NES's cubic stiffness term, $k_{b}$ is the stiffness coefficient of the base, $x$ is the displacement of the NES, and $x_{b}$ is the displacement of the base. As a result of the pure cubic (nonlinearizable) nonlinearity of the spring, the response of the NES lacks a preferential resonance frequency, and can thus resonate with, and transfer energy from, any mode of the base structure to itself in a nearly one-way fashion and locally dissipate it without backscattering [5]. In contrast to the Type VI NESs, the Type I NESs are energized at a later phase of the structural response, as they are designed to absorb and dissipate blast energy that is scattered from the Type-VI NESs, and also to dissipate the energies of torsional modes of the base structure (actually, due to the nearly symmetric blast excitation of the base structure, the excitation of such torsional modes ended up being minimal). As shown in [14], this type of NES with smooth nonlinearity is "activated" only once the energy of the structural mode with which it engages in resonance capture exceeds a certain energy threshold; this is in contrast to the Type VI NES which operates right from the beginning (in fact, during the first cycle) of the response. 


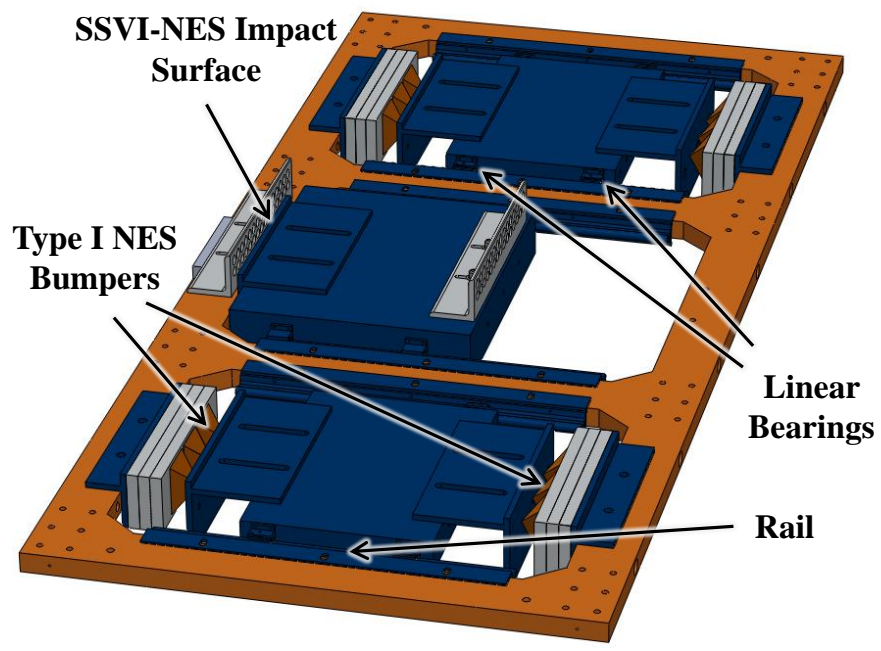

(a)

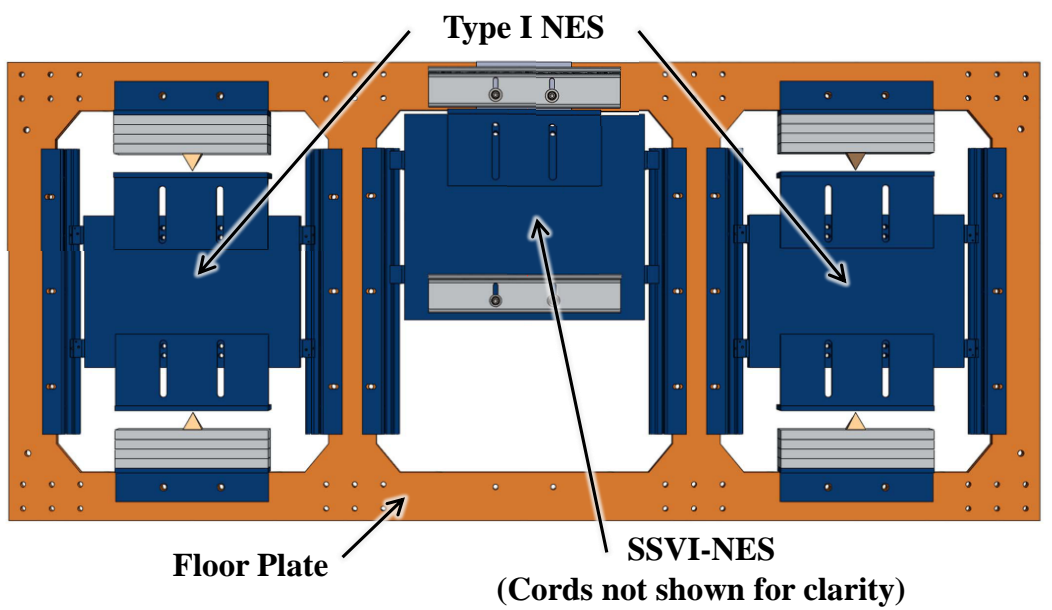

(b)

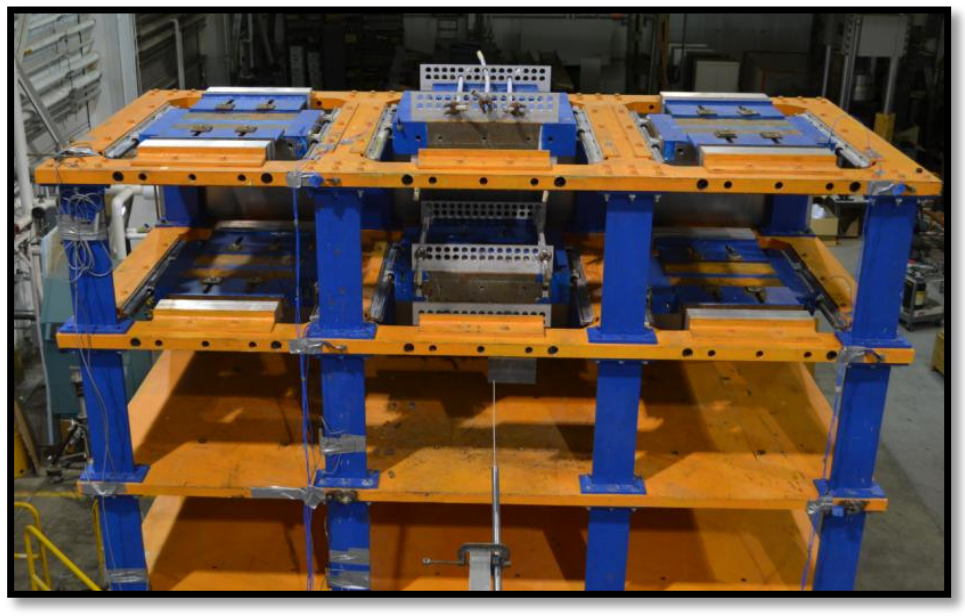

(c) 183 (b) schematics for the eighth and ninth floors, and (c) physical realization of the system of NESs on the base structure. 
Type I NES

(a)
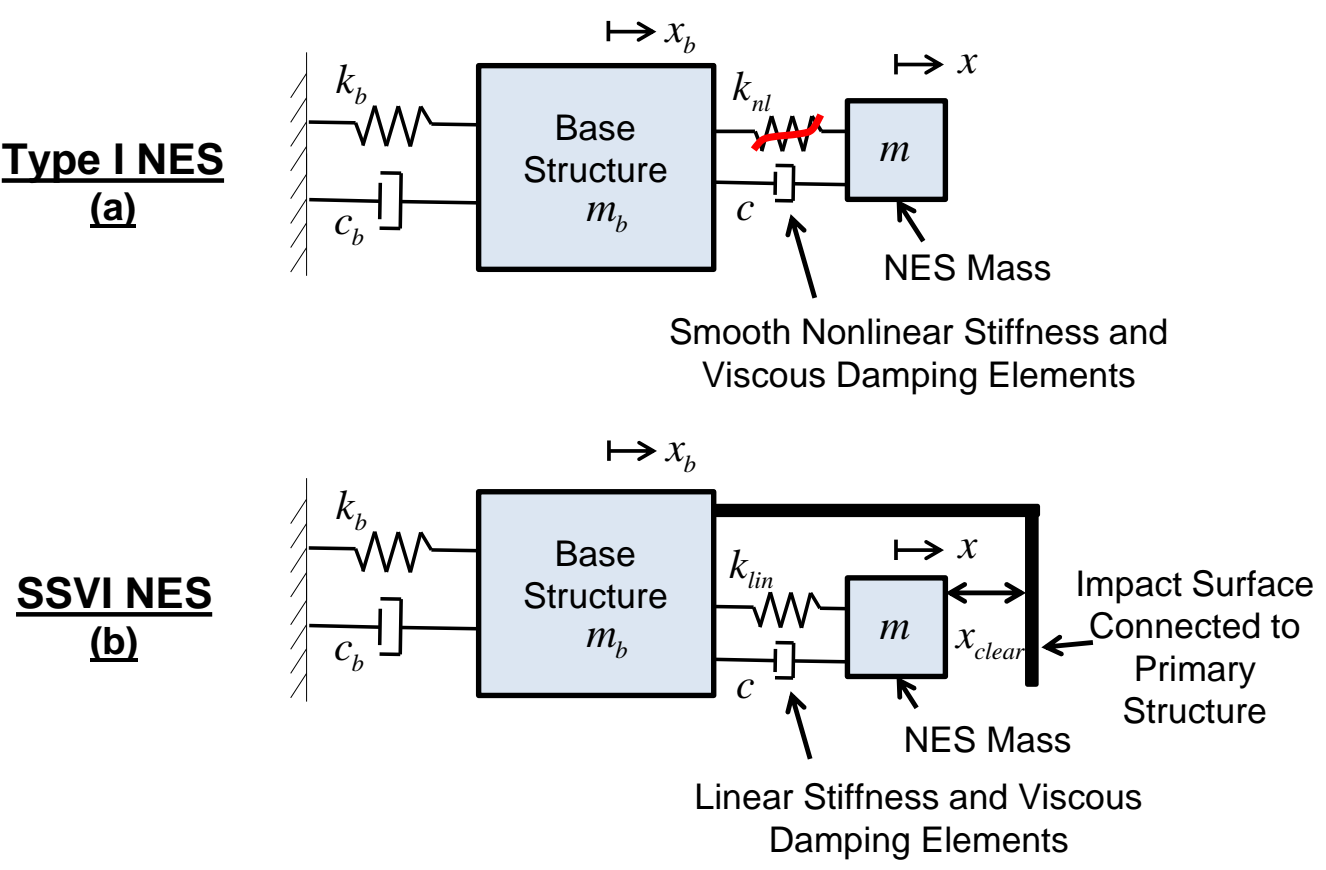

Figure 3. Conceptual models of (a) Type I and (b) Type VI (SSVI) NESs connected to a linear singledegree-of-freedom oscillator.

In their physical realizations, shown in Figure 2, the NESs consist of solid steel masses with Thomson SSUPB012 pillow blocks containing linear bearings mounted on opposite sides. These linear bearings allow the masses to move freely on sets of $1.91 \mathrm{~cm}$ round rails attached to the floor plate inside each cutout. The rails permit the NES mass to move parallel to the short direction of the floor plate, in the direction of the loading. Each of the NESs in this system incorporates a locking mechanism; consequently, tests can be performed with the NESs locked (prevented from moving relative to the floor) or unlocked (free to move).

The Type VI (SSVI) NESs are centered in both the eighth and ninth floors. Each is coupled to the floor plate through a set of elastic cords which are positioned such that, at rest, the NES mass is in contact with one side of the floor plate (see Figure 2a,b). There is a small pretension in the elastic cords so that the NES mass is secured against the side of the floor plate under very low loading conditions. When the motion of the floor is large enough to exceed this small force, the NES moves relative to the floor. The elastic cords connecting the NES to the floor plate stretch and produce a substantial restoring force that propels the NES mass into the floor with significant velocity. The result of this steel on steel collision is energy dissipation (the amount of which is related to the incoming velocity and coefficient of restitution) and high-frequency scattering of the remaining energy. 
The side-mounted NESs on the eighth and ninth floors are of Type I. These NESs are coupled to the structure using specially shaped elastomeric bumpers mounted on each end of the NES mass (see Figure 2a,b), so that one or the other is placed in compression when the NES moves. The special shape of these bumpers imparts an essentially nonlinear, approximately cubic restoring force to the NES mass [3,17]. Each Type I NES installed on the ninth floor uses a single bumper, while three different elastomeric bumpers are used in each Type I NES in the eighth floor. The reason for this discrepancy is that the ninth floor NESs are expected to engage in more intense resonance captures with the structural modes compared with the eighth floor NESs since they undergo larger displacements and, hence, exhibit more pronounced nonlinear effects.

\subsection{Computational Model Devolvement}

Modeling of the integrated system considered in this paper has been discussed in $[3,18]$; however, as computational models will be used in the system optimization discussed in this section and in comparisons presented in subsequent sections of this work, an overview of the development of the computational model of this system is warranted here. This computational model is based on the differential equations which describe the dynamics of the combined base structure and system of nonlinear energy sinks. The total combined system model has a total of 24 degrees-of-freedom. The base structure is modeled as a linear system with nine degrees-offreedom describing its translational motion and nine degrees-of-freedom describing its torsional motion. More information on the model of the base structure can be found in section 2.3.1. Mathematical models for the Type I NES and Type VI NES present in the combined system can be found in section 2.2. These NES are modeled as directly coupled to the floor mass they are physically located at and account for six degrees-of-freedom.

\subsubsection{Base structure}

A separate phase of the experimental testing of this system took place at the US Army Engineering Research and Development Center, Construction Engineering Research Laboratory (CERL) in Champaign, Illinois and utilized a large shake table known as the Triaxial Earthquake and Shock Simulator (TESS) [19]. With this shake table, a sine sweep ground motion was used to excite the base structure for the purpose of system identification. For this test, the NESs were locked, and the structure was assumed to be linear. The acceleration response of the structure to this sine sweep was collected at each floor, primarily using PCB model 353B33 accelerometers. The results of this test were post-processed in the frequency domain and peak-picking was used to extract the natural frequencies and mode shapes of the structure in its weak direction, which is the direction in which the blast would primarily excite the structure. The identified natural frequencies of the structure in the weak direction of the motion were 1.74, 5.38, 9.10, 12.72, 15.96, 18.95, 21.63, 23.92, and 25.48 Hz.

Initial modeling of the base structure was performed with a finite element model in which the structure was composed of beam and shell elements. This model was then updated with the 
experimentally obtained system identification results to closely reproduce the built structure's natural frequencies and mode shapes. As a lower-order model of the base structure was desired, a static condensation strategy was used to extract from this higher-order model a nine degree-offreedom lumped mass model associated with the weak direction translational motion of the structure. This low-order model corresponds well with the experimentally measured properties of the base structure, with the relative error between the natural frequencies of the nine degreeof-freedom model and the experimentally identified ones being $5.5 \%$ or less for each mode. Similarly, the experimental system identification efforts identified the torsional natural frequencies and modes of the structure. These results were then used to produce a separate nine degree-of-freedom lumped mass model associated with the torsional response of the structure. However, as the loading, base structure, and system of NESs are generally symmetric, the experimentally observed torsional component of the response was minimal for the blast tests and, therefore, not excited in the majority of simulations presented in this paper.

2.3.2 Analysis of the integrated model of the base structure with NESs attached

A system of differential equations for the integrated base structure/NES system was constructed by coupling the linear model of the base structure and the strongly nonlinear models of the NESs. Simulations with this model were performed using a fourth/fifth order RungaKutta method [20], which was modified to consider the vibro-impacts at the sites of the Type VI NESs (SSVI). In particular, when an impact was detected within the simulated time step, the time at which the impact occurred was precisely located and used to interpolate the state of the system. The impact was then modeled by applying a coefficient of restitution, while conserving linear momentum to calculate the post-impact state before restarting the otherwise smooth simulation of the transient dynamics of the integrated system.

In modeling this system, the simulations considered the impulse produced by the blast via a change in the initial velocity of the base structure degrees-of-freedom. This simplification is justifiable because the time-scales of the vibration of the structure are much slower than the duration of the blast; consequently, the global vibration of the structure can be considered a function of the total impulse produced by the blast instead of the specifics of the blast pressure time-history.

\subsubsection{NES parameter optimization}

Using the computational base structure/NES system model, an optimization procedure was employed to select the target stiffness and damping coefficients and impact-surface clearances of the physical system of NESs, given preselected NES masses [18]. The primary goal of this optimization was to maximize the first mode effective damping measure [5], which indicates the intensity of the effective energy "dissipation" of the first bending mode during the response to the blast; we note that, in addition to the energy dissipated by the damping of that mode, this measure includes the energy transferred from the mode to higher frequency modes due to the strong nonlinearities. The effective damping of the first bending mode is defined as 


$$
\lambda_{e, B 1}=-\frac{\int_{0}^{\infty} \frac{\mathrm{d}}{\mathrm{dt}}\left(\left\langle\dot{q}^{2}(t)\right\rangle\right) d t}{\int_{0}^{\infty}\left\langle\dot{q}^{2}(t)\right\rangle d t}
$$

283 where $\dot{q}(t)$ is the modal velocity of the first bending mode and $\left\langle\dot{q}^{2}(t)\right\rangle$ is defined as the 284 envelope of the expression $\dot{q}^{2}(t)$. The maximization of this measure was chosen as the 285 objective function for the optimization of the structural response because it provides a numerical 286 measure of the apparent energy loss of the lowest frequency (and highest-amplitude) structural 287 mode with consideration of the energy transferred to the other structural modes due to the 288 nonlinear effects of the NESs.

Although the primary goal of the design-optimization process was to achieve a design that was effective for eliminating energy from the first bending mode of the structure, it was important to address the potential effects of excited torsional modes in experimental blast tests. To that end, the design of the NES parameters was performed in two stages of optimization. In the first stage, parameters of the two identical Type-I NESs coupled to the eighth floor were selected by maximizing the effective damping of the first torsional mode of the nine-story structure using MATLAB's gradient-based optimization program fmincon [21]. The objective function for this stage was evaluated based on the response of the structure to an impulsive torque similar to that caused by a 12 psi-ms blast with $1.0 \mathrm{~m}$ eccentricity. Bending modes were not excited during this stage of the optimization due to the symmetry of the system and the loading, and the other NESs - two Type-VI NESs and two, ninth-floor Type-I NESs-were assumed to be locked (i.e., their masses were included, but motion relative to the structure was not). The result of this stage of the design optimization was a pair of identical Type-I NESs that were well suited to interact with the first torsional mode should it become excited during experiments.

Design parameters of the two Type-VI NESs and the ninth-floor Type-I NESs were selected in the second stage of the design by maximizing the effective damping of the first bending mode over a range of simulated blast intensities. Impulsive excitations of varying intensity and zero eccentricity were used in simulations, and the objective function approximated the integral of the effective damping of the first bending mode over a range of initial conditions. If $\mathbf{P}_{\mathbf{0}}$ is an impulsive force vector equivalent to a 30 psi-ms blast, then the intensity of the excitation can be scaled by

$$
\mathbf{P}_{j}=s_{j} \mathbf{P}_{\mathbf{0}}
$$


to approximate similar blasts. By simulating responses of the system to $\mathbf{P}_{j}$ for $j=1,2, \ldots, N$ and then evaluating the effective damping of the first bending mode $\lambda_{e, \mathrm{~B} 1}$, the objective function can be computed as

$$
f_{\mathrm{obj}}=\Delta s \sum_{j=1}^{N}\left(\lambda_{e, \mathrm{~B} 1}\right)_{j}
$$

where $\Delta s=s_{j+1}-s_{j}$ for $j=1,2, \ldots, N-1$ and $s_{j+1}>s_{j}$. Optimization of the design parameters in this stage was performed using MATLAB's genetic algorithm, ga, and refined using the gradient-based fmincon. This approach was selected over exclusive use of fmincon because the genetic algorithm is better suited for locating the global maximum when there are multiple local maxima, and fmincon is better suited for finding a local maximum with high precision.

It was critical that optimization of the two Type-VI NESs and the ninth-floor Type-I NESs be performed simultaneously in order to take advantage of potential synergistic effects between NESs of both types. Simultaneous optimization resulted in undamped type-VI NESs with a coefficient of restitution of one (i.e., perfectly elastic impacts). This somewhat surprising result for an application which seeks to maximize dissipation is due to the interaction between, and combined effects of the Type-I and type-VI NESs. Subsequent parameter optimization runs enforced a lower limit of $500 \mathrm{Ns} / \mathrm{m}$ on the NES viscous damping coefficients and an upper limit of 0.8 on the coefficient of restitution. These values were estimated based on observations of previous experimental hardware.

In the second stage of the design process, NES parameters were optimized to maximize the effective damping of the weak-direction first bending mode when the structure was subjected to impulsive loads equivalent to blast excitations - according to Eq. (5) - over one of three different intensity ranges: 3-18 psi-ms, 18-39 psi-ms, and 39-60 psi-ms. Designs resulting from the medium- and high-intensity excitation ranges had similar effectiveness over the entire range $(0$ 60 psi-ms), but the design from the high-intensity excitation range was selected for its more consistent performance. The resulting NES parameters are listed in Table 1, where negative values for the clearance between impacting surfaces indicate that impacts should occur on the side opposite the direction of the excitation. As expected from the previous discussion, the viscous damping coefficients of the type-VI NESs are nearly equal to the minimum allowable, and the coefficient of restitution is the maximum allowable. 
Table 1. List of optimized NES parameters; viscous damping coefficient $c$, linear stiffness coefficient

\begin{tabular}{|c|c|c|c|}
\hline \multicolumn{2}{|c|}{ Eighth Floor } & \multicolumn{2}{|c|}{ Ninth Floor } \\
\hline Type-VI & & Type-VI & \\
\hline$k_{\text {lin }}$ & $14546 \mathrm{~N} / \mathrm{m}$ & $k_{\text {lin }}$ & $12219 \mathrm{~N} / \mathrm{m}$ \\
\hline$c$ & $503 \mathrm{Ns} / \mathrm{m}$ & $c$ & $573 \mathrm{Ns} / \mathrm{m}$ \\
\hline Clearance & $-1.5 \mathrm{~mm}$ & Clearance & $-1.5 \mathrm{~mm}$ \\
\hline \multicolumn{2}{|c|}{ Type-I (Identical Pair) } & \multicolumn{2}{|c|}{ Type-I (Identical Pair) } \\
\hline$k_{\mathrm{nl}}$ & $7.56 \times 10^{8} \mathrm{~N} / \mathrm{m}^{3}$ & $k_{\mathrm{nl}}$ & $1.07 \times 10^{8} \mathrm{~N} / \mathrm{m}^{3}$ \\
\hline$c$ & $721 \mathrm{Ns} / \mathrm{m}$ & $c$ & $2439 \mathrm{Ns} / \mathrm{m}$ \\
\hline
\end{tabular}

The performance of the system with optimized NESs is shown in Figure 4 and compared to the effective damping with some or all of the NESs locked (i.e., NES motion relative to the structure is restrained). Effective damping of the first bending mode of the system when all of the NESs are locked is shown as the dashed line close to zero (i.e., the structure is lightly damped). When the Type-I NESs are unlocked and the type-VI NESs are locked, effective damping is increased to approximately $0.5 \mathrm{~s}^{-1}$ for most excitations and greater than $1.0 \mathrm{~s}^{-1}$ for excitations equivalent to a 6 psi-ms blast. When the Type-I NESs are locked and the type-VI NESs are unlocked, the effective damping is greater than $2 \mathrm{~s}^{-1}$ for equivalent blasts greater than 5 psi-ms. Effective damping for the system with all NESs unlocked is more than $4 \mathrm{~s}^{-1}$ excitations greater than 10 psi-ms - significantly better than all other configurations.

It is important to note that the effects of the Type-I and type-VI NESs are not additive (i.e., they are not independent of one another). Consider, for example, the effective damping of the system excited by a 30 psi-ms blast. When only the Type-I NESs are unlocked, the effective damping is around $0.6 \mathrm{~s}^{-1}$. It improves to approximately $2.4 \mathrm{~s}^{-1}$ when only the Type-VI NESs are unlocked. If the effects of the Type-I and Type-VI NESs were independent of one another, we would anticipate that the effective damping of the system with all NESs unlocked would be around $3 \mathrm{~s}^{-1}$; however, simulation of the system response indicates that the effective damping is greater than $4 \mathrm{~s}^{-1}$. Furthermore, Figure 4 shows that this improvement in effectiveness occurs despite fewer impacts from the type-VI NESs. 

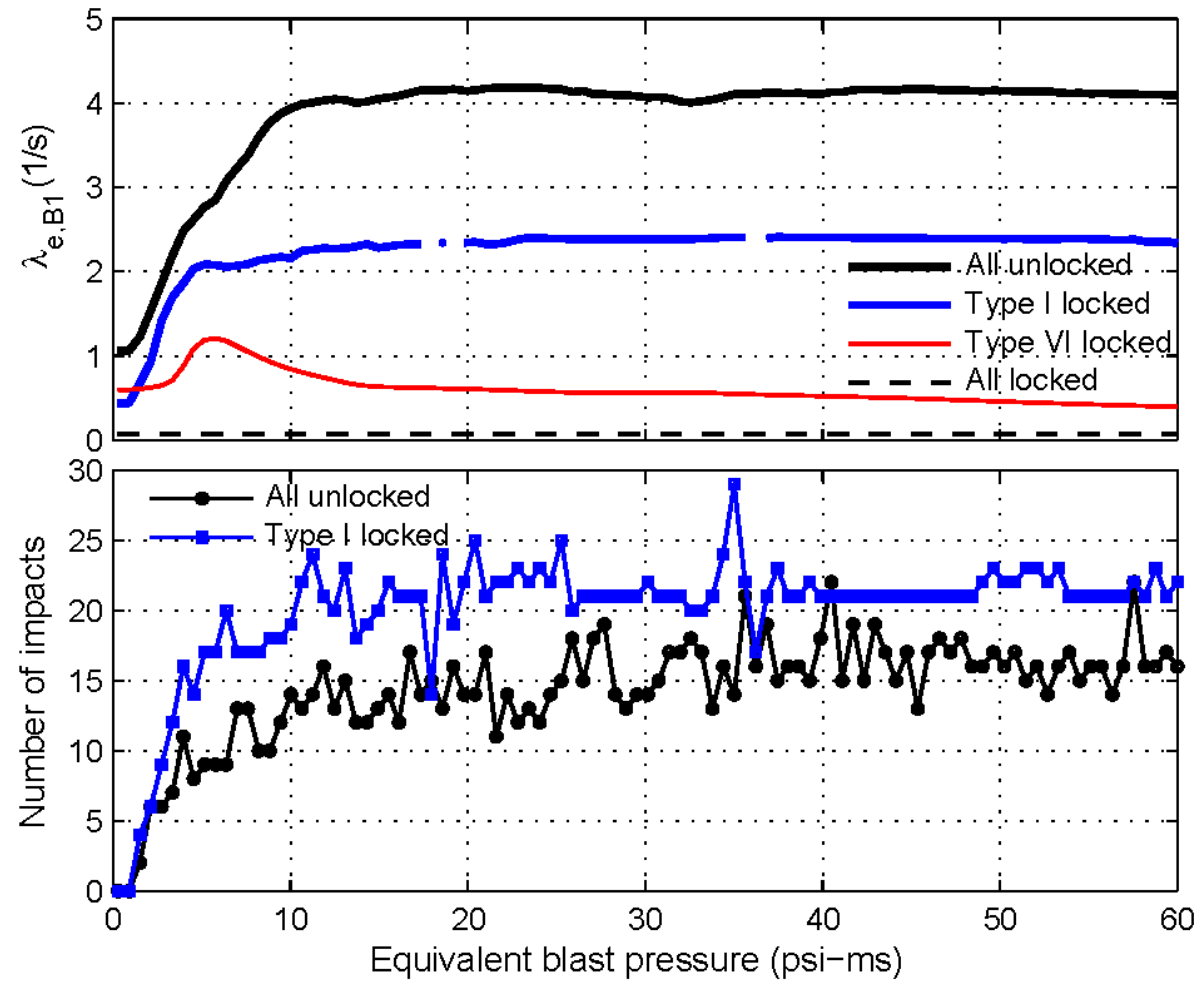

Figure 4 Simulated effective damping of the first bending mode of the system optimized for a range of blast intensities, and the number of Type-VI NES impacts during four seconds of simulated response.

Synergistic effects between the Type-I and Type-VI NESs are responsible for the improvement in performance when all of the NESs are free to oscillate. By designing Type-I and Type-VI NESs simultaneously, they assume complementary energy-management roles. The stiffness of the Type-I NESs is too large to encourage TET with the first bending mode for the energy levels that are considered in this design. However, Type-VI NES impacts create coupling between the low- and high-frequency modes of the system, allowing energy to be quickly transferred to higher-frequency modes. The higher-frequency modes dissipate energy more quickly than the fundamental mode, as evidenced by the effective damping of the system with only the type-VI NESs unlocked. Furthermore, stiff Type-I NESs readily engage in TET with the higher-frequency modes and quickly dissipate energy. In summary, Type-VI NESs take on the task of redistributing energy via impacts and Type-I NESs dissipate energy by engaging in TET with higher-frequency modes. In fact, as mentioned in the previous discussion, optimized typeVI NESs will have no mechanism for dissipation if the viscous damping coefficient and coefficient of restitution are not constrained to reflect realistic limits; therefore, the ideal type-VI NES in this application is only capable of redistributing energy. 


\subsubsection{Realized NES parameters}

The parameters of the NESs that resulted from this optimization analysis were then used to synthesize the design of the physical system of NESs. Table 2 summarizes the estimated parameters of the physically realized system of NESs. It is important to note that although this physical system of NESs has been used in a separate study related to the use of NESs to mitigating the response of structures subjected to seismic ground excitation [3], this system of NESs was optimized and realized specifically for the blast loading featured in this study.

The stiffness parameters listed in Table 2 were estimated using component-level experiments, including uniaxial tests of the Type VI (SSVI) NESs' elastic cords and the Type I NESs' elastomeric bumpers. Furthermore, the NES damping parameters were estimated by correlating experimental data and computational simulations.

As shown in Table 2, the mass ratios of the NESs are relatively high compared with commonly utilized values in linear absorbers. The nonparasitic design of the system negates the weight penalty; additionally, the performance and robustness of the system under uncertainty, particularly over extended time, justifies the additional complexity introduced by the highly nonlinear nature of the NESs.

The load deformation curves for bumpers which have the same designs as those utilized in the Type I NESs are shown in Figure 5. These load deformation curves were experimentally obtained from the component-level uniaxial tests. Further details on the design and fabrication of elastomeric bumpers to realize a desired nonlinear stiffness profile can be found in [17].

Unlike the stiffness values, comparison of Table 1 and Table 2 shows that the estimated damping coefficients of the NES physical realizations do not match up well with the damping values derived from the optimization study. While the physical design of the NES, for instance the shape of the elastomeric bumpers, correspond well with the stiffness of these devices, the connection between the physical design and the damping is less straightforward. While the damping of the NESs are modeled as viscous damping, no physical viscous dampers exist in the NESs. Rather, the damping of the NESs comes from multiple different sources, including the material damping of the bumpers and the friction in the linear bearings. The estimated viscous damping coefficients attempt to capture the net effect of all these dissipative sources. While the departure of the measured damping from the optimized damping values is not ideal, it is acceptable because the NES types used in this work are generally less sensitive to the level of NES damping once a minimum level of damping has been achieved [7,22]. 


\begin{tabular}{c|cccc}
\hline & $\begin{array}{c}\text { Mass (\% of Total } \\
\text { Mass of the } \\
\text { Structure })\end{array}$ & $\begin{array}{c}\text { Stiffness } \\
\text { Coefficient }\end{array}$ & $\begin{array}{c}\text { Stiffness } \\
\text { Exponent }\end{array}$ & $\begin{array}{c}\text { Damping } \\
\text { Coefficient }\end{array}$ \\
\hline $\begin{array}{c}\text { Eighth Floor } \\
\text { Type I NES }\end{array}$ & 1.5 & $1.5 \times 10^{9} \mathrm{~N} / \mathrm{m}^{3}$ & 3 & $100 \mathrm{~N}-\mathrm{s} / \mathrm{m}$ \\
\hline $\begin{array}{c}\text { Ninth Floor } \\
\text { Type I NES }\end{array}$ & 1.5 & $2.0 \times 10^{8} \mathrm{~N} / \mathrm{m}^{3}$ & 3 & $138.5 \mathrm{~N}-\mathrm{s} / \mathrm{m}$ \\
\hline $\begin{array}{c}\text { Eighth Floor } \\
\text { SSVI NES }\end{array}$ & 3.5 & $15,000 \mathrm{~N} / \mathrm{m}$ & 1 & $50 \mathrm{~N}-\mathrm{s} / \mathrm{m}$ \\
$\begin{array}{c}\text { Ninth Floor } \\
\text { SSVI NES }\end{array}$ & 3.5 & $12,000 \mathrm{~N} / \mathrm{m}$ & 1 & $50 \mathrm{~N}-\mathrm{s} / \mathrm{m}$ \\
\hline
\end{tabular}
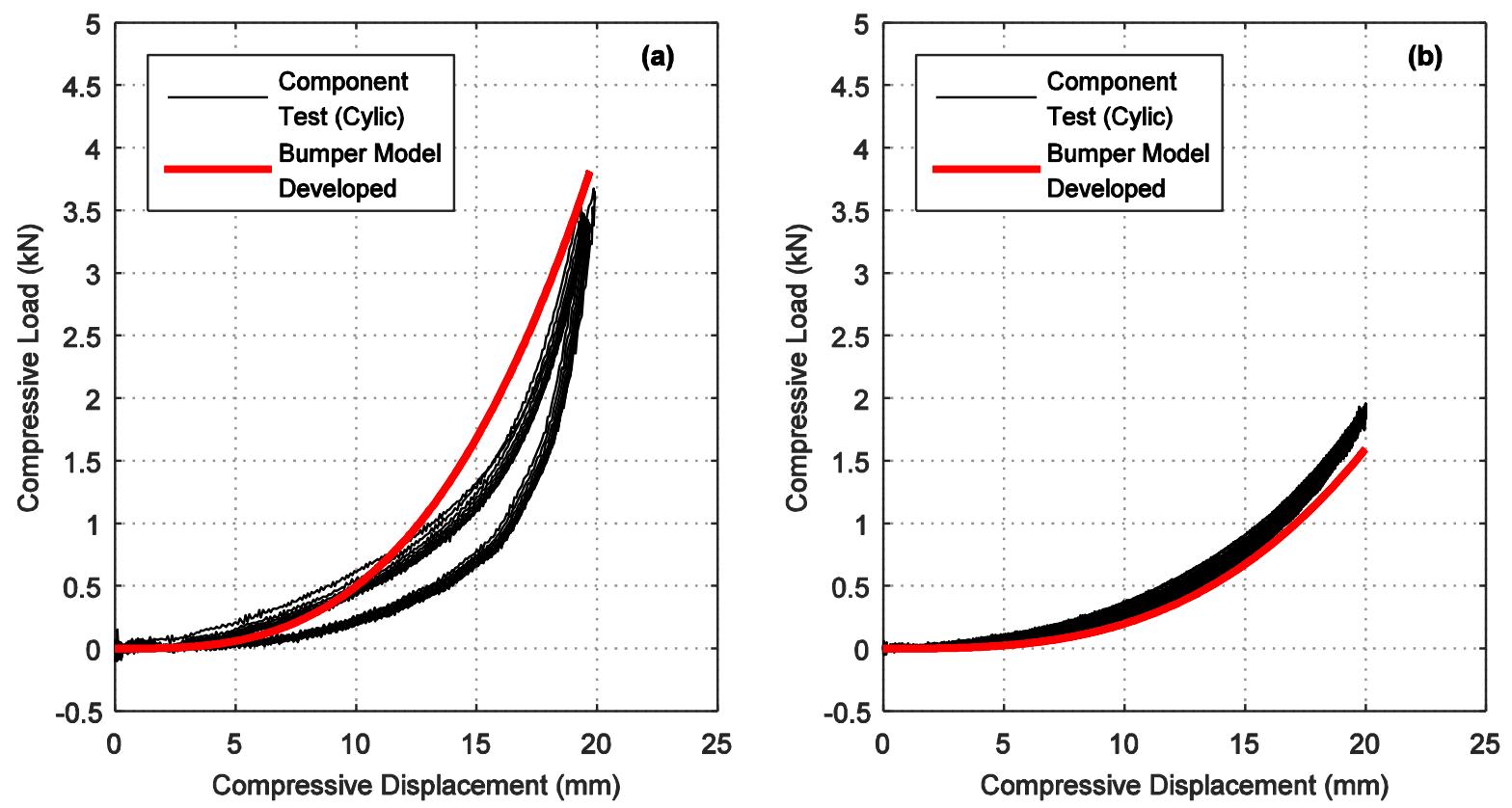

Figure 5. Load deformation curves for elastomeric bumpers utilized in (a) eighth floor Type I NESs and (b) ninth floor Type I NESs.

\section{Test Setup}

In this section, the experimental setup for the blast testing is presented. This includes a discussion of the blast load configuration and the instrumentation utilized to measure the response of the base structure and system of NESs. 


\subsection{Blast configuration}

Blast testing of the structure studied in this work was performed at the US Army Corps of Engineers Big Black Test Site. This testing range is affiliated with and located near the US Army Corps of Engineers Engineer Research and Development Center in Vicksburg, Mississippi. Tests at this site were performed using air bursts of the high explosive C-4. For these tests the base structure was oriented such that its cladding faced toward the center of the blast. With this orientation, the majority of the energy introduced into the base structure from the blast resulted in the motion of the structure in its weak direction, as intended.

The objective of this testing was to investigate the ability of the system of NESs to rapidly attenuate the response of the base structure due to a general offset blast loading at various intensities. As a result of this objective, the goal of the charge designs was not to simulate specific blast profiles that would occur in particular scenarios, but rather to produce a more general blast load profile, resulting in a nearly uniform pressure impulse on the face of the cladding, as much as possible. When designing the charges to accomplish this goal, the variables considered were the standoff distance (the horizontal distance of the charge from the structure), the height of the charge relative to the ground, and the amount of explosive utilized (as measured by the weight of the charge). In general, when the standoff distance is increased, the uniformity of the pressure load on the structure also increases; however, with increased standoff distance, the pressure and resulting impulse on the structure are reduced. Consequently, if the standoff distance of a blast is increased, to maintain the same total average impulse on the structure the charge weight must also be increased.

Accounting for the variables of the charge configuration, the constraints on charge size due to restrictions imposed by the test site, and the geometry of the base structure, the software UrbanFx, an extension of the BlastX software published by the US Army Corps of Engineers [23], was used to simulate the effects of the blast on the structure. With these simulations and desired levels of average pressure impulse, the three charge configurations shown in Table 3 were produced. The moderate level blast response is of primary interest for this work, and thus the corresponding configuration will be referred to from now on as the primary charge configuration.

As seen from Table 3, the standoff distance of the blast in each of these configurations is quite small. Because of a limitation regarding the charge size at the test site, this small standoff distance was necessary to provide the desired level of impulse. Additionally, this table also shows that the low-intensity and primary charge configurations feature one explosive charge positioned roughly at the mid-height of the structure; however, the high-intensity configuration, which produces the largest average impulse on the structure, is designed by combining three separate simultaneous charges. The reason for separating the charge along the height of the structure is the large charge weight required to achieve the desired average pressure impulse; 
Table 3. Charge configurations

\begin{tabular}{ccccc}
\hline & $\begin{array}{c}\text { Average Predicted } \\
\text { Pressure Impulse } \\
\text { (kPa-msec) }\end{array}$ & $\begin{array}{c}\text { Standoff } \\
(\mathbf{c m})\end{array}$ & $\begin{array}{c}\text { Height of } \\
\text { Burst }(\mathbf{c m})\end{array}$ & $\begin{array}{c}\text { C-4 Charge } \\
\text { Weight }(\mathbf{k g})\end{array}$ \\
\hline $\begin{array}{c}\text { Low-Intensity Charge } \\
\text { Configuration }\end{array}$ & 106 & 213 & 295 & 0.54 \\
\hline $\begin{array}{c}\text { Primary Charge } \\
\text { Configuration }\end{array}$ & 209 & 213 & 295 & 1.40 \\
\hline $\begin{array}{c}\text { High-Intensity Charge } \\
\text { Configuration }\end{array}$ & 620 & 173 & $\begin{array}{c}76,295, \\
404\end{array}$ & $\begin{array}{c}1.27,1.27, \\
1.27\end{array}$ \\
\hline
\end{tabular}

In addition to calculating the average pressure impulse on the structure, the analysis with BlastFx provides the pressure impulse at points along the structure's cladding face. A contour plot of the pressure impulse on the cladding of the base structure for the primary blast configuration listed in Table 3 is shown in Figure 6. This contour plot shows that, while the design goal of the blast was to provide a uniform pressure impulse on the face of the structure, the resulting charge configurations deviate significantly from uniformity. Nonetheless, this charge configuration is adequate for testing since, while not perfectly uniform, the pressure impulse is spread across the cladding and will excite the global vibration modes of the base structure. 


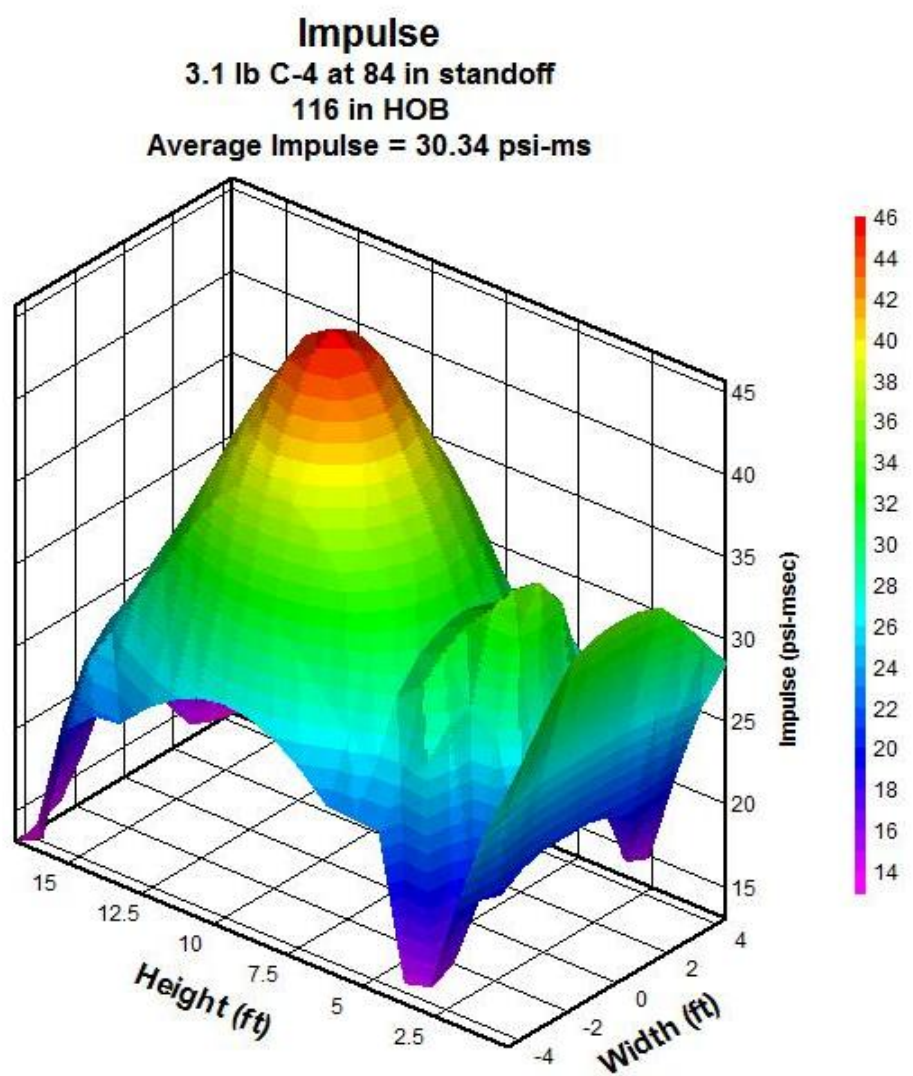

Figure 6. Computationally predicted cladding pressure impulse contour plot for the $209 \mathrm{kPa}-\mathrm{msec}$ primary charge configuration.

To produce the standoff distances and heights of burst for the charge configurations shown in Table 3, a system of rigging towers and cords was used to suspend the charges. Adjustable lengths of cord were attached to the charge and strung between two of the rigging towers to control the height of the burst, while an additional cord attached to the charge and a third tower were used to control the standoff distance. The rigging towers, which can be partially seen in Figure 1, were constructed of steel tubing, had a heavy base and a small profile, which allowed them to survive the blast undamaged. The final component of the charge configuration, the weight of the charge, was simple to produce as the pliability of the C-4 made it straightforward to shape it into a sphere of the required charge weight.

\subsection{Instrumentation}

A variety of instrumentation was utilized to record the loading and response of the base structure and the system of NESs during blast testing, including accelerometers, pressure gages, strain gages, and displacement transducers. The accelerometers used for this testing were PCB model 353B33, Endevco model 7264-200, and Endevco model 7264-2000. The majority of these accelerometers were oriented to measure the response of the base structure in the direction of the blast. Accordingly, accelerometers were placed on every floor of the structure and on each 
of the NESs. At locations of anticipated high acceleration, such as the SSVI NESs and the floors with NESs, the accelerometer models with higher maximum acceleration capacity were utilized. In addition to acceleration, direct displacement measurements were obtained with displacement transducers. Due to equipment availability and practical limitations on the height of the transducer mounting fixtures, displacement measurements of only the first and third floors were collected.

To measure the demand on the first story, several first floor columns were instrumented with YEFLA-5-5LT strain gages manufactured by the Tokyo Sokki Kenkyujo Co. These gages were placed at mid-width of the columns and positioned $7.6 \mathrm{~cm}$ from the columns' end. This position allowed the strain gage to measure the strain resulting from the motion of the structure in the direction of the blast.

To measure the loading from the blast, the structure was instrumented with multiple pressure gages. These gages were attached to mounts directly connected to floor plates of the structure. In order to measure the pressure on the face of the cladding, the heads of the gages extended out of small holes in the cladding and were held in place with mounting brackets.

\section{Experimental Results}

Using each of the primary and low-intensity charge configurations outlined in Table 3 , the structure was tested twice: first with the system of NESs unlocked (free to move, inducing strongly nonlinear effects in the structural response) and then with the NESs locked (prevented from moving, thus just adding a mass effect to the linear structural dynamics). For the high intensity charge configuration a single test of the structure with the NESs unlocked was performed, since the intensity of the blast damaged the cladding, so no meaningful comparison with the corresponding case with the NESs locked could be performed.

In Figure 7, a single frame from the high speed video of the blast using the primary charge configuration is presented. As this frame was taken only milliseconds after the blast was initiated, the NESs, which are contained in the top two floors of the structure (and unlocked for this test), have not yet had time to move significantly. This frame demonstrates that the pressure wave resulting from the blast causes significant deformation to the cladding. Despite this deformation, after each test using either the low-intensity or primary charge configurations, the cladding remained largely intact and, after minimal repairs, was ready to transfer the load of the next blast to the structure. However, after the blast with the high-intensity charge configuration, the cladding was significantly damaged (it was partially ripped apart), and this signified the end of the experimental tests. 


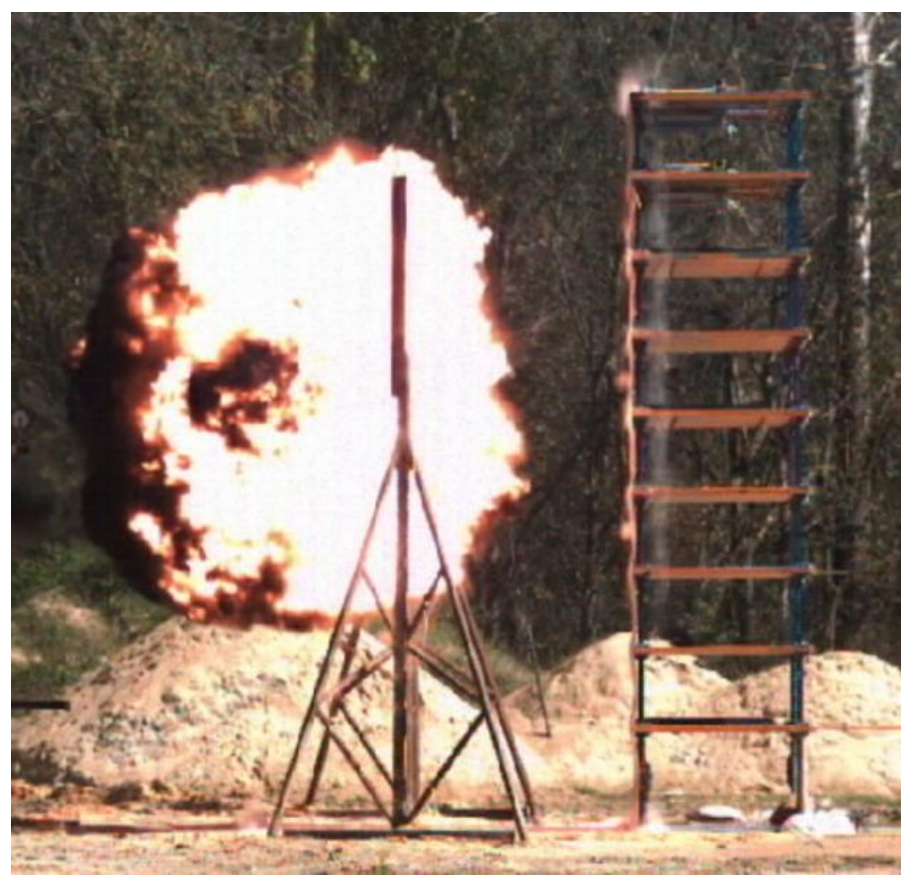

Figure 7. Base structure during blast with primary blast configuration.

\subsection{Response to primary charge configuration (209 kPa-msec blast)}

Pressure measurements from the blast taken from gages on the cladding during the tests utilizing the primary blast configuration are shown in Figure 8. The pressure gages used to obtain this figure are mounted on the fourth and seventh floors. These results show that the pressure time-histories have a nearly instantaneous jump in positive pressure which occurs at the arrival of the blast pressure wave. After the positive pressure decays, suction of air back towards the center of the blast results in lower amplitude and longer duration negative phase of the pressure. The shape observed in these results corresponds well with the expected theoretical blast pressure time-history from literature [2,24]. As Figure 8 shows, the gages on the fourth floor measure a much higher peak pressure than those on the seventh floor. This pressure distribution is logical as the gages on the seventh floor are further from the origin of the blast than those on the fourth floor. Aside from differences in peak pressure, this figure also shows a time lag in the pressure measurements from the gages on separate floors. Once again, this is logical due to the proximity of the respective gages to the blast origin. In addition to the characteristics of the blast pressure at different points along the cladding, Figure 8 demonstrates that the pressure loading on the structure for both tests is nearly equivalent, as the measurements from the NESs locked and unlocked tests show very good agreement. This agreement is important as it indicates the response of the structure from the two blast tests corresponding to NESs unlocked and locked can be accurately compared. This enables us to directly assess the efficacy of our design based on nonlinear targeted energy transfer for rapidly and effectively mitigating the structural response due to the applied blast loads. Similarly, a comparable set of pressure measurements were obtained from the results of the low-intensity blast tests. 


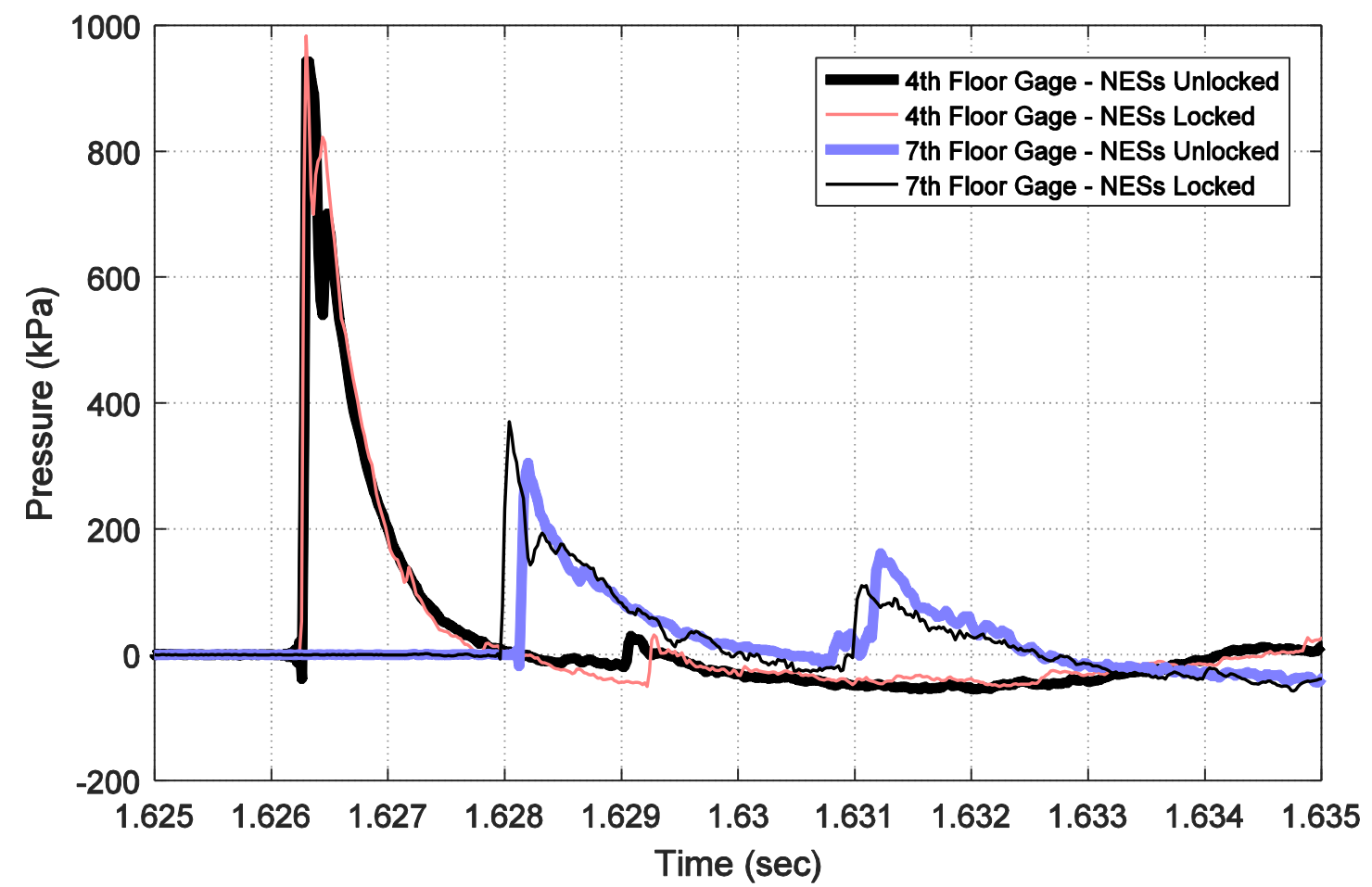

Figure 8. Pressure time history during tests utilizing the primary blast configuration, measured at the fourth and seventh floors at the center of the cladding.

In Figure 9, the third floor displacements measured during the primary charge configuration tests are plotted; additionally, this figure shows simulation results that will be discussed in section 4.4. As this figure shows, when the NESs are unlocked the response of the structure quickly decays due to rapid nonlinear low-to-high frequency scattering of the blast energy; however, when the NESs are locked, the motion of the structure persists for a significantly longer time. Additionally, this figure shows that, along with rapidly eliminating the motion, with the NESs unlocked the peak displacement at this floor level is significantly reduced. The reduction observed in the positive direction is relatively modest $(20.2 \%)$, but the reduction in the negative direction is quite substantial $(52.9 \%)$. This demonstrates that the strongly nonlinear action of the Type VI NESs are affecting the structural response from the first cycle (especially the second half of that cycle), and proves the efficacy of the nonlinear targeted energy transfer concept. The rapid scattering of blast energy to the higher modes of the structure (which will be discussed below) results in immediate and drastic reduction of the overall structural response and more effective dissipation of the energy by better "utilization" of the inherent structural damping of the structure itself. Equivalently, the actions of the NESs lead to an effective nonlinear redistribution of the blast energy within the modal space and paves the way for better "activation" of the inherent structural damping capacity. This provides an extra benefit toward the blast mitigation objective, in addition to the portion of the blast energy that is dissipated by the dissipative elements of the NESs themselves due to the relative motion of all of the NESs and 
the inelastic collisions of the SSVI NESs. This contrasts with the linear structural response in Figure 9 (with the NESs locked) where the blast energy is confined mainly to the first mode which possesses the lowest frequency and the highest amplitude. Moreover, the effective damping of the blast energy in that case is drastically lower compared to the nonlinear case.

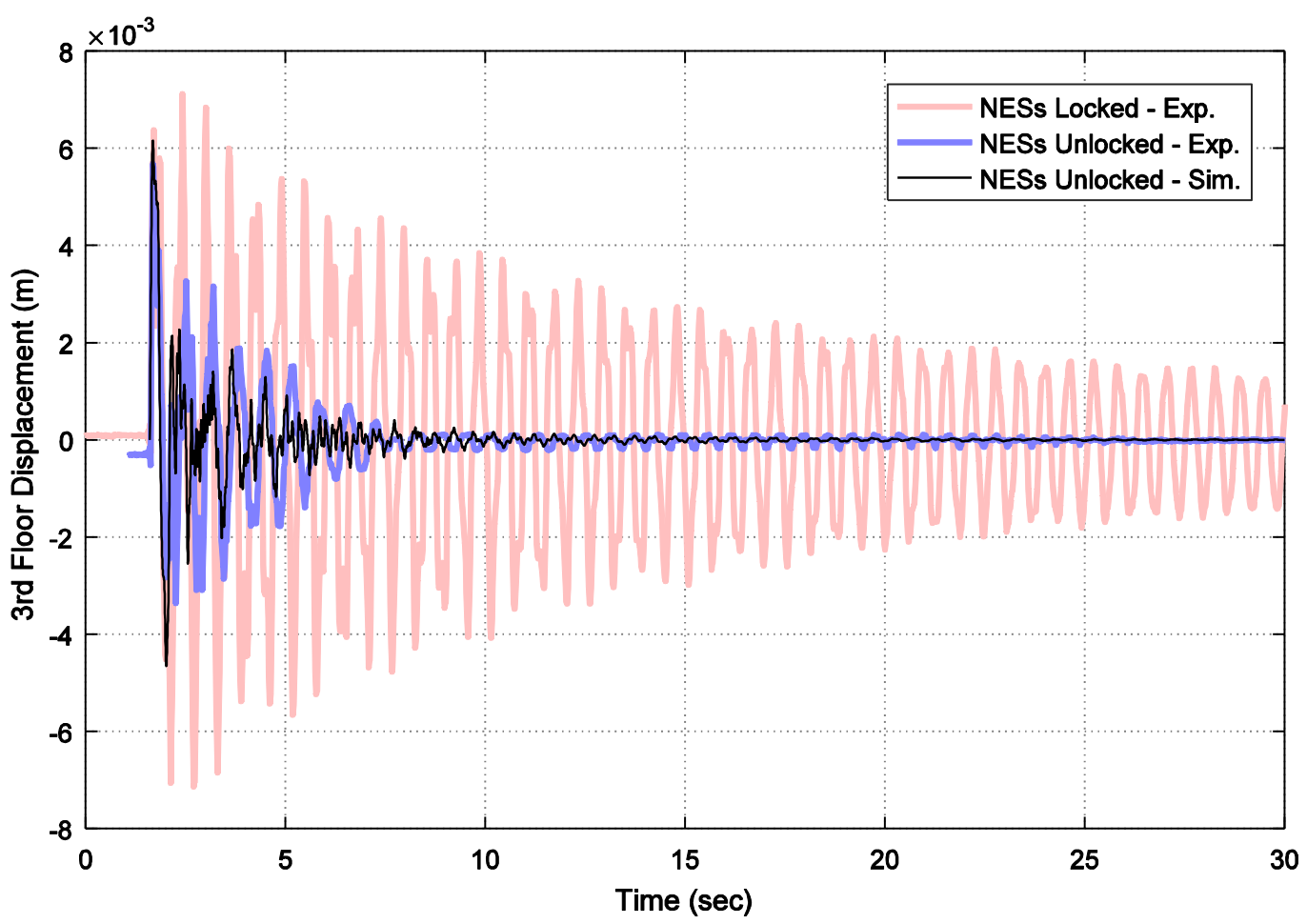

Figure 9. Third floor displacement response to primary blast configuration.

Figure 10a shows the wavelet spectra of the acceleration response of the seventh floor to the blast tests when the NESs are locked and unlocked. The wavelet spectrum can be a useful tool for examining the behavior of the structure because it is capable of showing changes in the frequency content of the structure's response over time [25]. As Figure 10 shows, when the NESs are locked, the seventh floor acceleration response of the structure due to the blast is spread over most of the modes of the structure in the weak translational direction; however, the most significant, and prolonged, motion is observed in the lowest three modes. When the NESs are unlocked, Figure 10 shows that motions in nearly all the modes of the structure decay faster than the case when the NESs are locked; this is especially true for the lowest modes of the structure which decay very rapidly when the NESs are unlocked. Furthermore, as the input from the blasts was comparable for the two tests, this figure suggests the transfer of energy to the higher modes of the structure due to motion of the NESs as these wavelet spectra show greater emphasis on higher modes of the structure's response when the NESs are unlocked. 

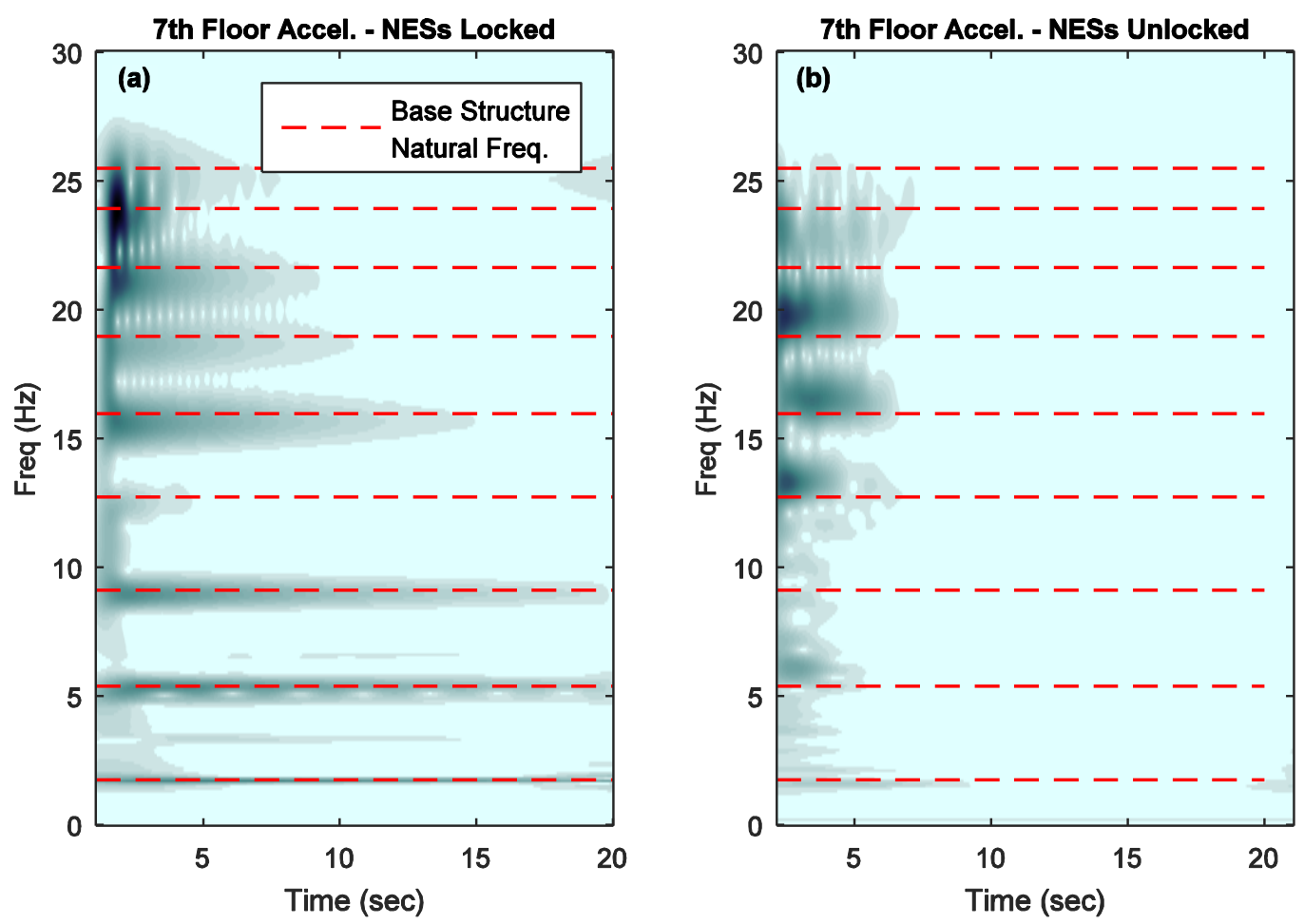

\section{8} 599

Figure 10. Wavelet spectra of the seventh floor acceleration response to primary blast configuration with (a) NESs locked and (b) NESs unlocked.

To better understand the strongly nonlinear action of the NESs in the responses shown in Figure 10, Figure 11 shows the time history and wavelet spectra of the acceleration response of the eighth floor NESs during the blast test for the case when they are unlocked. This figure shows that the NESs undergo large accelerations, but are only in motion (i.e., they are "activated") for a few seconds after the blast. During this time the frequency content of the response of the NESs is very broadband, as expected due to the essential nonlinearities in the restoring force the NESs are designed with. This broadband behavior is especially apparent during the impacts of the SSVI, which correspond to the peaks in the time history of the SSVI NES's acceleration and the corresponding heavily shaded bands in the wavelet spectra. This broadband behavior of the NESs is what allows them to couple the modes of the structure and engage in targeted energy transfer, rapidly scattering blast energy from low-to-high modes in a broadband fashion. Another noteworthy feature that can be observed from the wavelet spectra of the Type I NESs is the clearly defined smooth transitions in the wavelet spectra of the (smooth) Type I NESs as these NESs exit from their phases of large accelerations; these lower frequency transitions clearly appear in the wavelet transforms of the Type I NESs' blast responses in Figure 11. When viewed in appropriate frequency-energy planes, these transitions translate to the Type I NES dynamics following the backbone curves defined by the dynamics of the undamped Hamiltonian NES-structure system [22]. Once the blast energy is reduced and dissipated, the 
responses of the NESs decay as well, as the rapid blast mitigation objective is achieved. This explains the short periods of active NES operation in Figure 11.

Examining the resulting displacements and accelerations from the blast loading is useful for investigating the behavior of the structure; however, these measures do not directly correspond with the demand on the structure. To examine the demand on the structure, the strain measured at one of the interior first floor columns is shown in Figure 12. This strain is proportional to the column moment which results from the blast loading; thus, this measurement can be used to examine the primary source of structural demand on the first story, the location which is often the most critical part of a structure. Figure 12 shows that, like the displacement and acceleration responses previously examined, the strain in the first floor columns decays quickly when the NESs are unlocked. Additionally, with a reduction of $21.0 \%$ in measured peak strain compared to the locked case, this figure demonstrates that the passive system of NESs substantially reduces
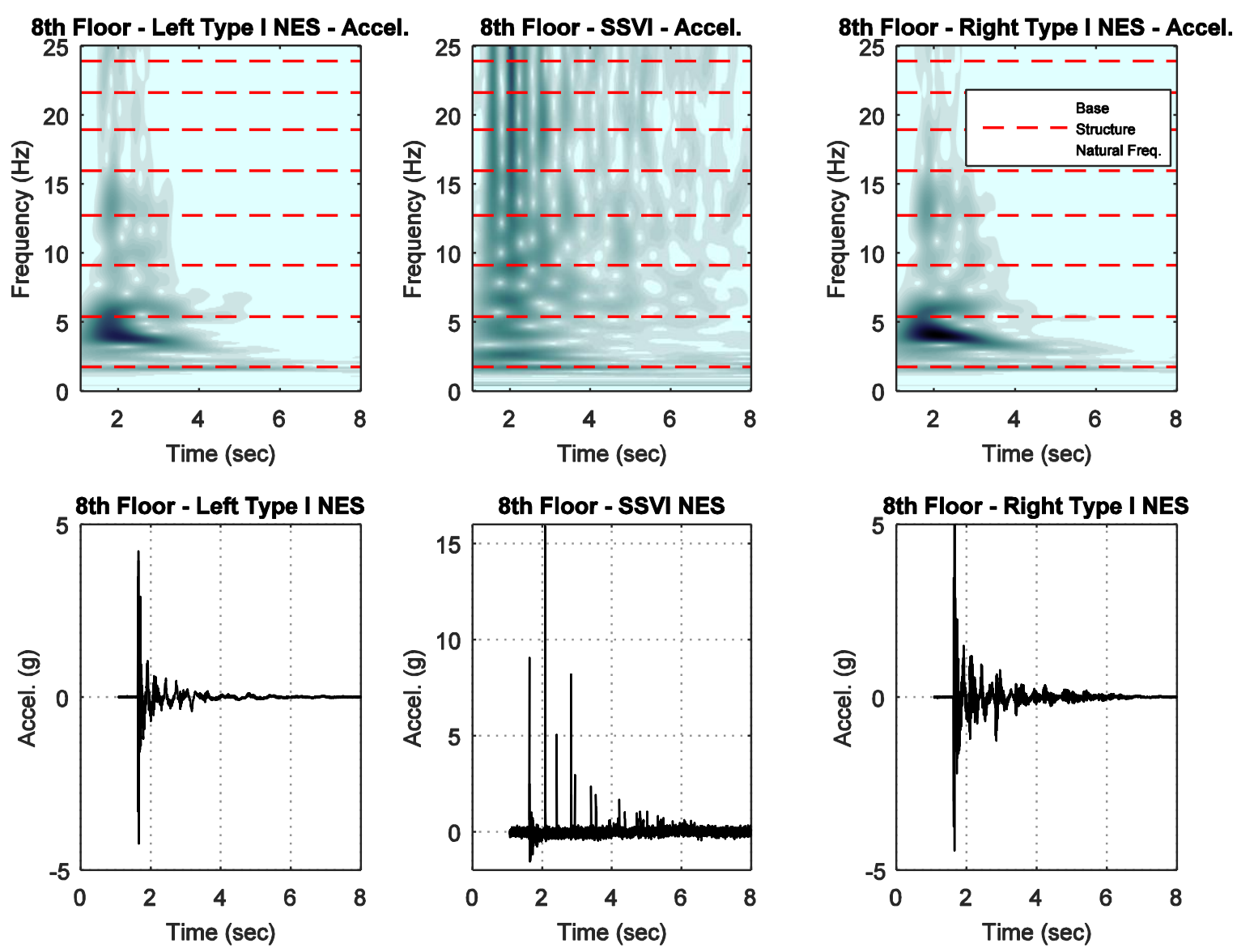

Figure 11. Eighth floor NESs acceleration responses to primary blast configuration - time histories and corresponding wavelet spectra. 


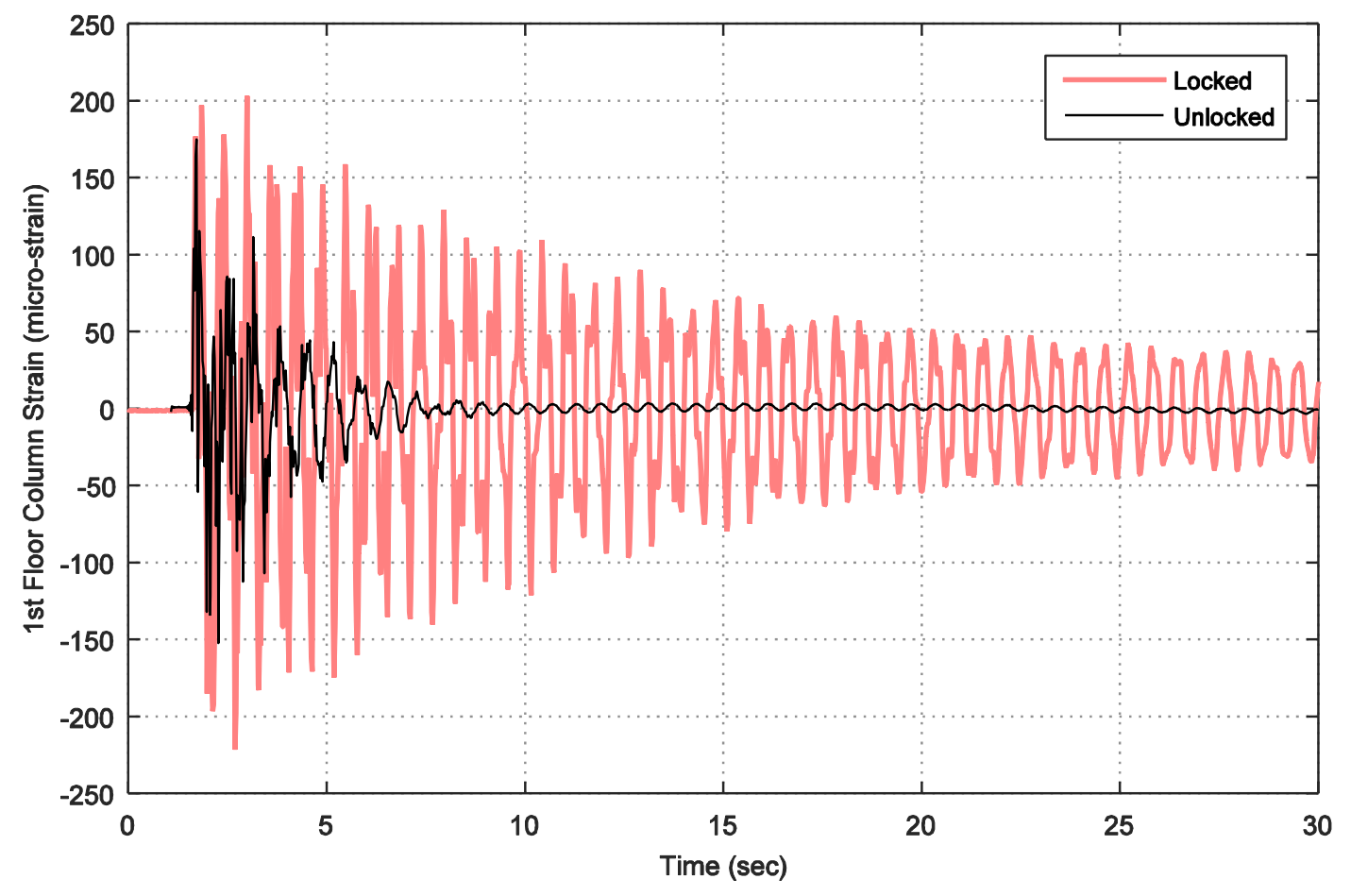

Figure 12. First floor column strain response to primary blast configuration.

\subsection{Response to the low-intensity charge configuration (106 kPa-msec blast)}

Using the low-intensity charge configuration outlined in Table 3 the structure was tested twice: with the NESs unlocked and with NESs locked. In Figure 13, the third floor displacements measured during the two tests are shown. Similar to the primary charge configuration considered in section 4.1, we note that when the NESs are unlocked, the response of the structure rapidly decays; however, when the NESs are locked, the motion of the structure persists for a significantly longer time. Additionally, this result demonstrates that, along with rapidly attenuating the motion, the peak structural displacement is reduced with the NESs unlocked. The reduction observed in the negative direction is relatively small $(5.6 \%)$, but the reduction in the positive direction is rather substantial $(24.1 \%)$. The change in the effectiveness of the NESs in mitigating the response of the structure seen here, is expected due to the energylevel dependency of this nonlinear passive mitigation system. Due to the relatively low displacements shown in this figure, an apparent irregularity is noticeable in the unlocked case a few seconds after the blast when the displacement is substantially reduced. This irregularity, which appears as a square wave in the displacement data, is purely numerical and occurs because the resolution limit of the displacement sensor has been reached, preventing the sensor from accurately measuring the small residual motion that persists at later times. 

(1) (1)

(1)

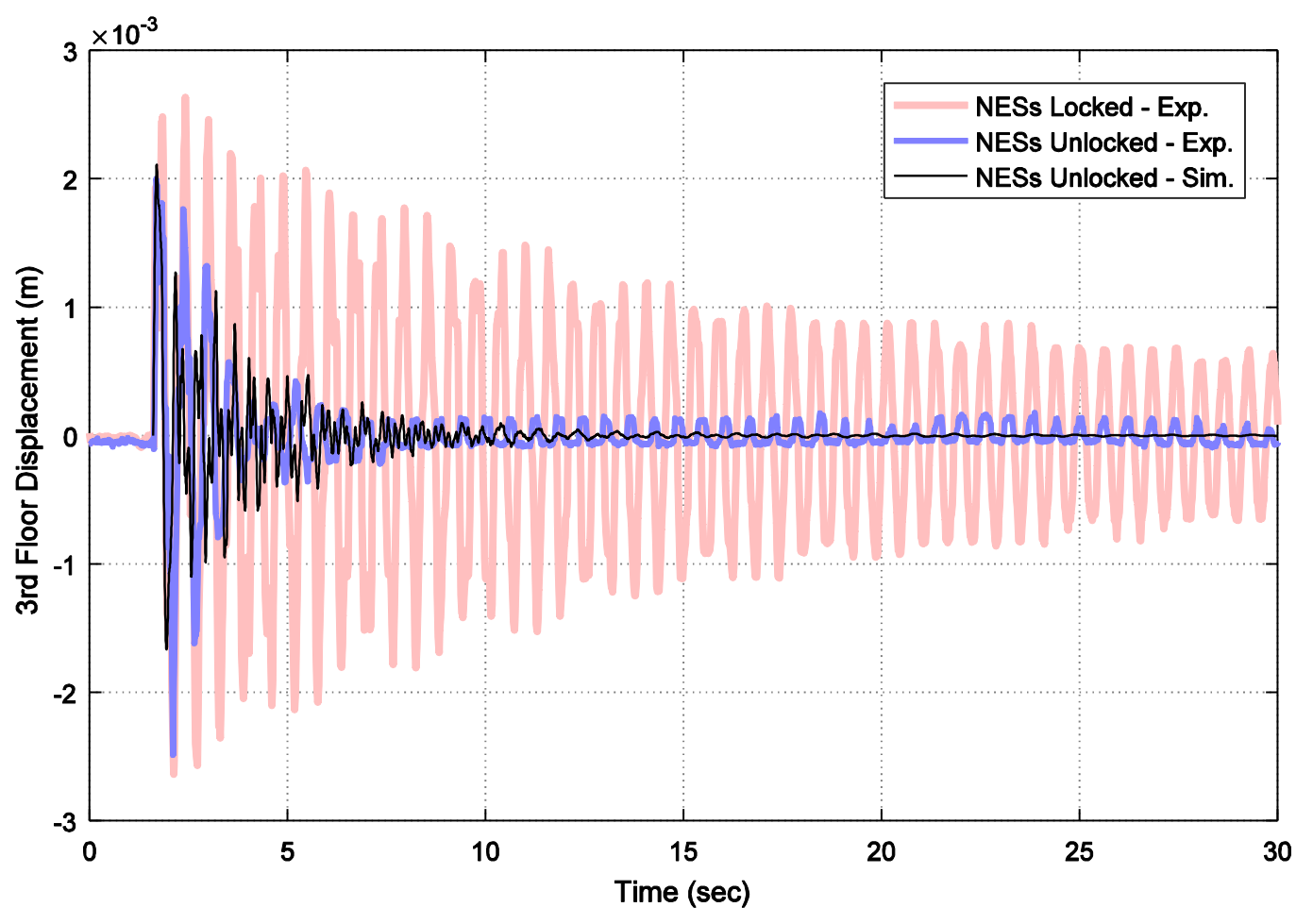

Figure 13. Third floor displacement response to the low-intensity blast.

To examine the demand on the first story, the strain measured at one of the interior first story columns is shown in Figure 14. Like the displacement response previously examined in 659 section 4.1, the strain in the first floor columns decays quickly when the NESs are unlocked.
660 Additionally, a reduction of $20.0 \%$ in the measured peak strain compared to the locked case is 661 observed with the system of NESs unlocked. 


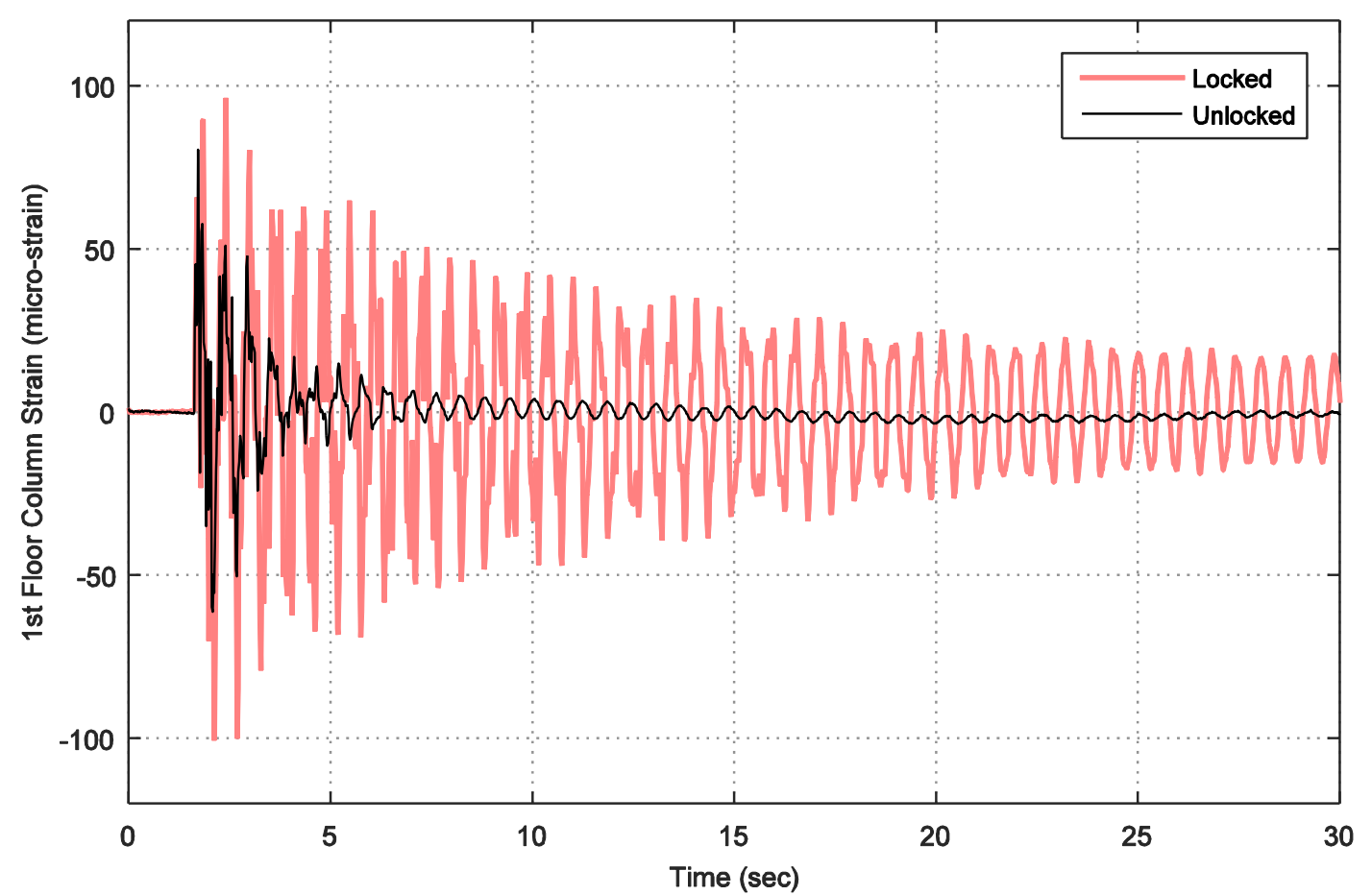

Figure 14. First floor column strain response to low-intensity blast

\subsection{Response to high-intensity charge configuration (620 kPa-msec blast)}

Using the high-intensity charge configuration outlined in Table 3 the structure was tested only once, that is, with the system of NESs unlocked. While a second test would ideally have been performed on the structure with the system of NESs locked, this test was not possible due to damage to the cladding sustained during the first blast with this charge configuration. Damage to the cladding in this test can be seen in Figure 15, which shows a single frame from the high speed video of this blast. Note that while the cladding was damaged in this test, the structural system of the base structure remained undamaged.

Clearly, without both corresponding tests with the NESs locked and unlocked, direct comparisons cannot be made to quantitatively determine the effectiveness of the system of NESs; however, the results of the unlocked test alone can be used in a qualitative manner to explore the performance of the system. 

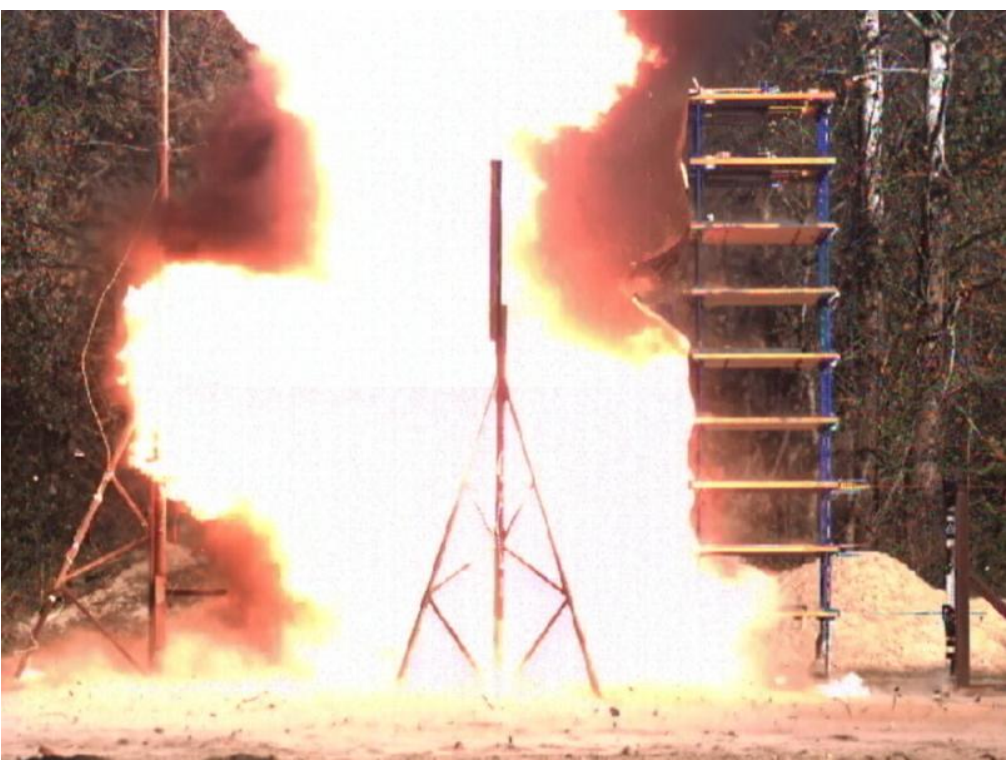

Figure 15. Base structure during blast with high-intensity blast configuration.

In Figure 16 the third floor displacement measured during this test is shown. As this figure shows, with the system of NESs unlocked, the response of the structure decays very rapidly. This rapid reduction is demonstrated by the fact that in about two cycles the amplitude of the displacements decreased by more than $80 \%$; compared to the previous experimental results, in the primary and low-intensity blast tests about three and five cycles, respectively, were necessary for that level of reduction to occur. This result was to be expected because the higher the blast intensity, the more energy is imparted to the base structure, and, as a result, the stronger the nonlinear effects due to action of the NESs become. In other words, for this highest blast level the nonlinear targeted energy transfers due to the NESs become more profound, so the attenuation of the structural response is faster and more significant compared to the lowerintensity blast experiments. This indicates that our NES-based design becomes increasingly more efficient for stronger blasts, which renders it especially suitable for mitigation of infrastructure damage under severe blast loading. 


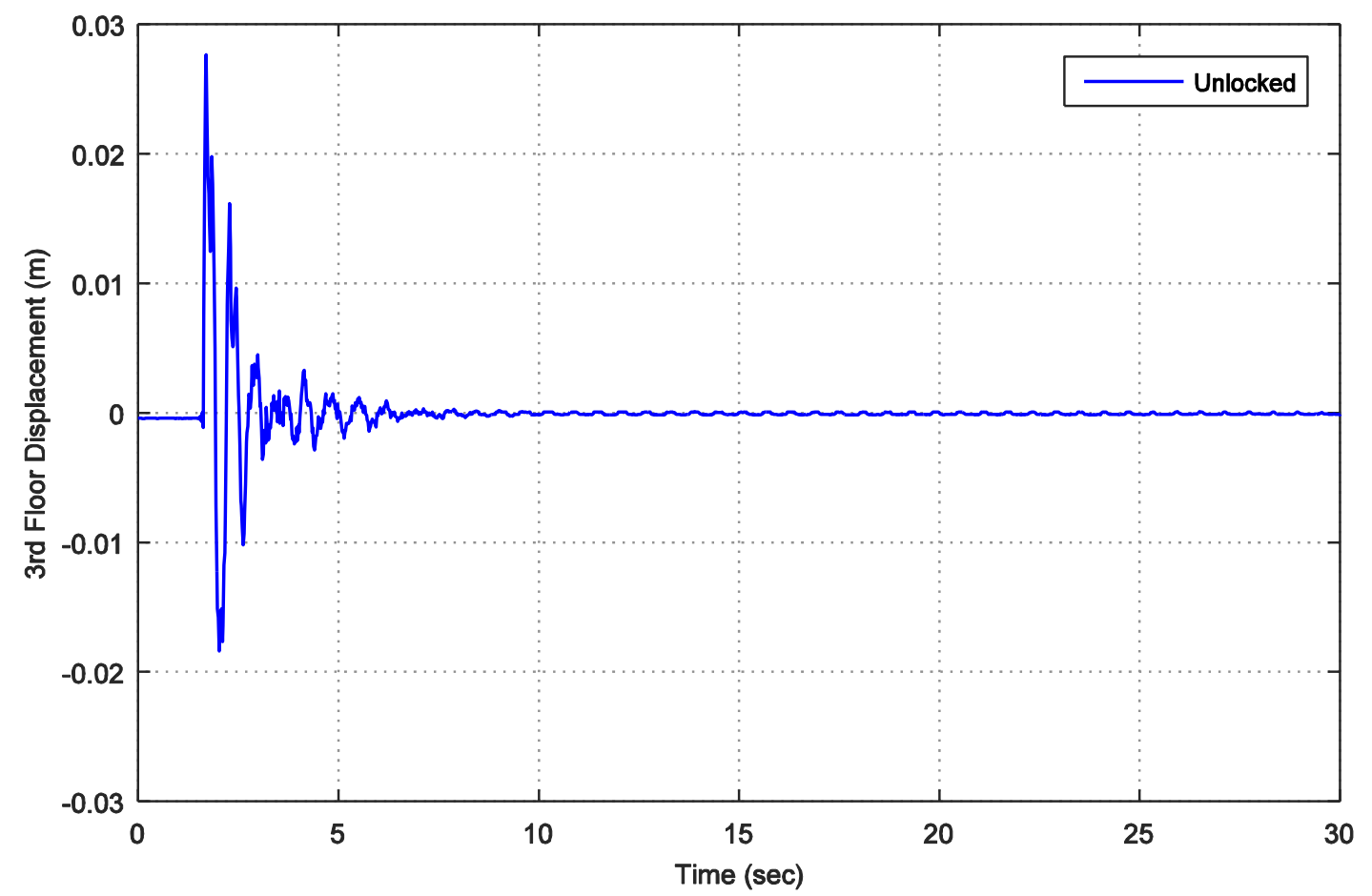

693

694

695

696

697

698

699

700

701

702

703

704

705

706

707

708

709

710

711

Figure 16. Third floor displacement response for the high-intensity blast configuration; note the faster response reduction compared to the previously examined more moderate blast configurations.

Figure 17 shows the wavelet spectrum of the acceleration response of the seventh floor to the blast test with the highest intensity $620 \mathrm{kPa}-\mathrm{msec}$ charge configuration. As similarly observed in the previously examined tests with the system of NESs unlocked, all the translational modes of vibration of the structure are attenuated a few seconds after the blast; however, the lower modes are attenuated especially quickly. Additionally, this figure shows that the response of the structure includes a large amount of activity of its higher modes. With no locked test to compare with, the possibility that this abundance of higher mode energy is solely a result of the blast cannot be precluded; however, previous results with the system of NESs locked and unlocked suggest that the abundance of higher mode energy is a consequence of nonlinear lowto-high frequency scattering of the blast energy by the system of NESs.

The strain measured at one of the interior first story columns during this test is shown in Figure 18. This figure shows that the strain in the first floor columns attenuates quickly when the NESs are unlocked with an $80 \%$ reduction in amplitude achieved in less than 1.5 seconds. Like the displacement measurement for this test shown in Figure 16, the attenuation in strain is faster than that observed in the lower level blast tests. The efficacy of the system of NESs in response to the high intensity blast, combined with the good performance observed at the other load levels, further demonstrates the robustness of the nonlinear design; i.e., that the system of 
4712 NESs examined is capable of providing rapid attenuation of the response of the structure across a 6713 wide range of load levels.

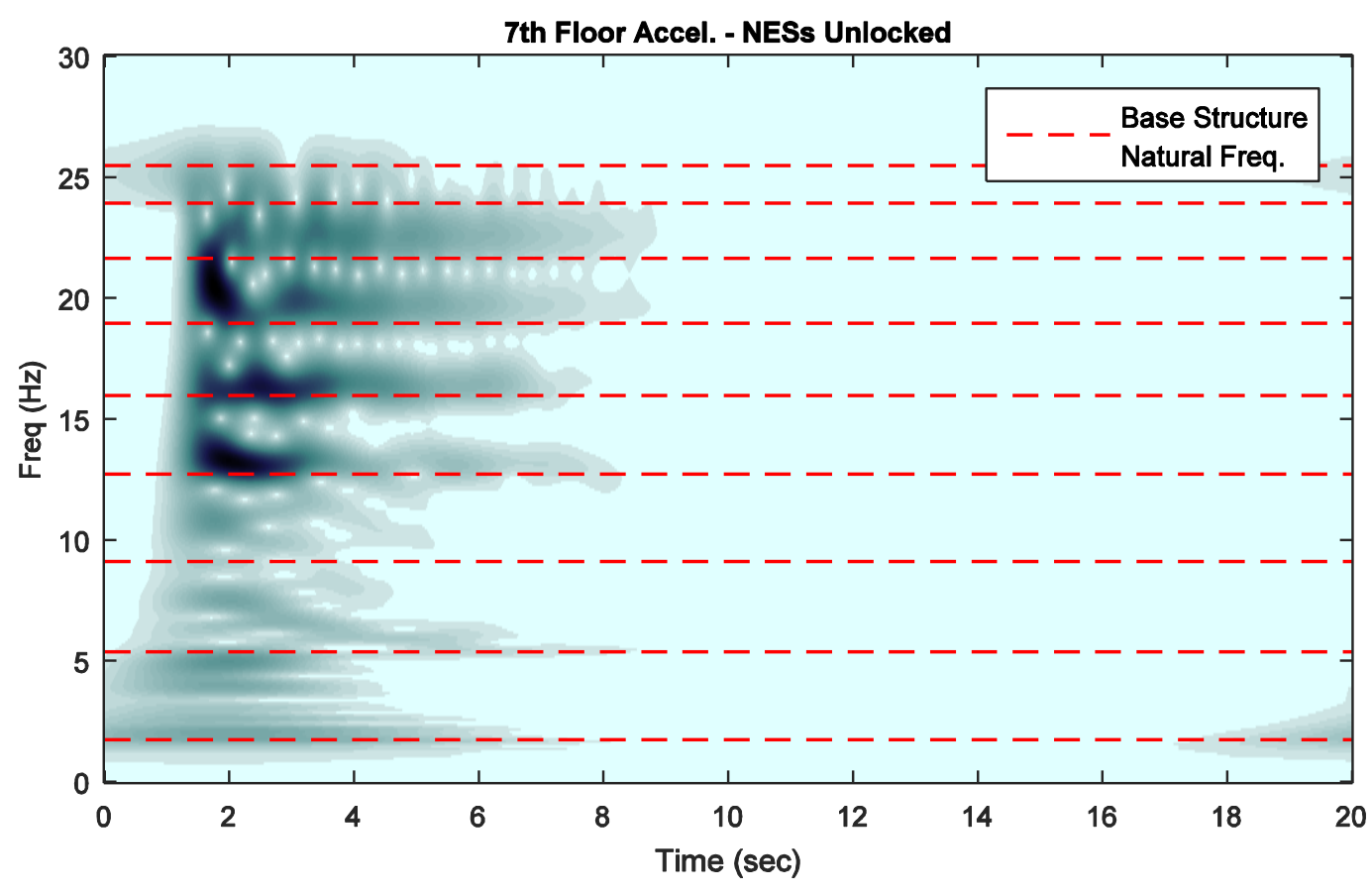

715 Figure 17. Wavelet spectrum of the seventh floor acceleration during the high-intensity blast with NESs 716 unlocked. 


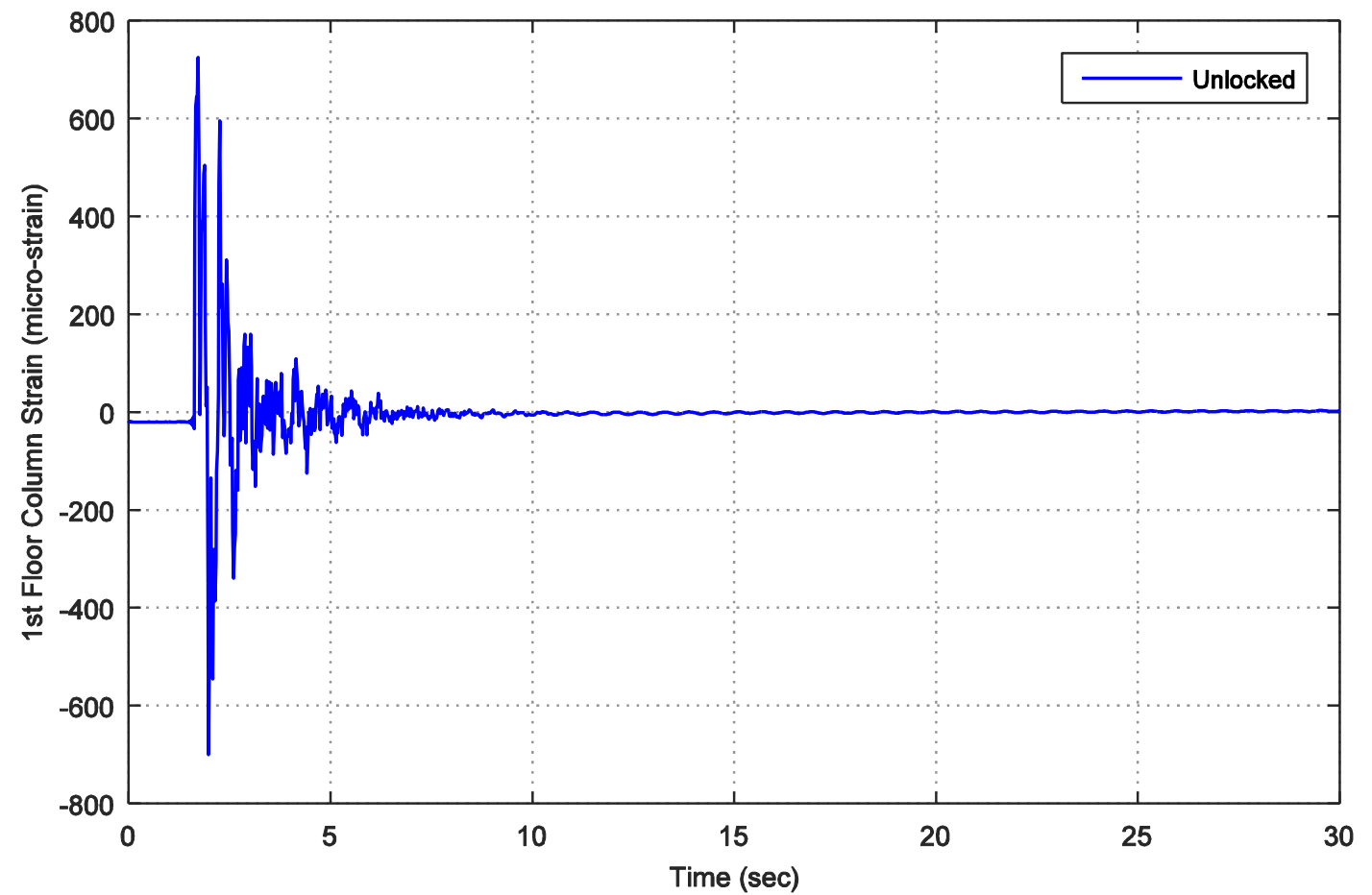

Figure 18. First floor column strain response for the high-intensity blast.

\subsection{Computational model validation}

Validation of the computational model of the base structure/NES system was performed using a comparison of the experimental response of the structure with unlocked NESs to the lowintensity and primary charge configurations and the simulated response.

In preparation for this comparison, several key features were extracted from the response of the experimental structure with the system of NESs unlocked. The first feature extracted was the effective loading on the structure due to each charge level. To obtain this information, the acceleration response of the base structure, which was experimentally measured at every floor, together with the displacement response, which was only measured at the first and third floors, were collected with the system of NESs locked. These acceleration and displacement time histories were then low-pass and high-pass filtered at $0.125 \mathrm{~Hz}$ and $100 \mathrm{~Hz}$, respectively. This filtering was performed to suppress high-frequency noise and remove non-physical lowfrequency drift in the signals. After low-pass and high-pass filtering, the signals were passed through a Kalman filter [26,27]. The Kalman filter uses the measured responses and the model of the base structure to estimate the velocity response of each story, which was not directly measured. Based on these measurements the velocity of each of the base structure's floors directly after the end of the each blast was extracted. 
The second feature extracted from the locked response was an estimate of the modal damping of the base structure. This feature was estimated by curve fitting the attenuated envelope of the modal acceleration response of each mode. The damping was estimated at each charge level due to possible changes due to damage to the cladding of the structure from the blasts.

Simulation of the response of the structure with the NESs unlocked was performed with the model introduced in section 2.3 incorporating the estimated base structure modal damping values. This system was loaded with the set of floor velocities estimated from the experimental results considered as initial velocities of the floors of the system. The resulting simulated displacement response of the third floor with the NESs unlocked is shown for the primary and low-intensity charge configurations in Figure 9 and Figure 13, respectively. These show that good agreement is observed between the simulation and the experiments with the NESs unlocked, both for the overall shape of the time series and the maximum and minimum peaks with an exception being the discrepancy in the minimum peak of the structural response for the low-intensity charge. This good agreement demonstrates the efficacy of the numerical simulations of the system and the underlying mathematical model.

The response of the structure to the high-intensity charge configuration was not utilized in the validation of the computational model. The reason for this is that no experimental tests of the structure with the system of NESs locked was performed at this load level. Due to the combination of damping from the NESs and the base structure when the NESs are unlocked, the response with the NESs locked is necessary for an estimation of the damping of the structure itself; this is particularly essential as the damage to the cladding likely increased the damping of the structure significantly.

\section{Simulations}

Using the model of the integrated base structure/NES system that was validated in the previous section, a computational study was undertaken to investigate the effectiveness of the system of NESs in controlling the response of the base structure when subjected to a broad range of impulsive loads of varying energy content. The impulsive load considered for this analysis was the uniform initial velocity of the base structure in its weak translational direction; i.e., the same primary direction as the blast loading experimentally studied in the last section. Different energy levels of this impulsive load were achieved by scaling the initial velocity. When analyzing the structure with the system of NESs unlocked, the initial velocity provided to the eighth and ninth floors of the base structure are scaled up by the square root of the ratio of the mass of each of the floors with and without the NESs. This scaling accounts for the difference in effective mass of the top floors when the NESs are locked and unlocked, and results in an equal amount of initial energy provided to the base structure when the NESs are locked and unlocked. For this analysis, one percent damping was assumed in each mode of the base structure. 
Figure 19 shows a plot of the percentage of energy remaining in the system one, two, and four seconds after the initial velocity (impulse) is applied to the base structure. These results were obtained from simulations for a wide range of initial velocities applied to the structure; alternatively, this can be thought of as a wide range of initial energy imparted to the structure. The energy in the system at the time instants examined was calculated by adding the kinetic and potential energies of the base structure to the kinetic and potential energies of the NESs. As the impulsive load was applied as an initial velocity on the base structure, the peak energy in the system was the initial kinetic energy in the base structure. This figure shows that, when the NESs are locked, the percentage of energy remaining in the system is not dependent on the initial energy level for the base structure alone; however, as expected, with the system of NESs unlocked, the performance of the system is dependent on the energy level imparted to it. For all three time instants investigated (namely, at one, two, and four seconds after the initial velocity is applied to the system), the percentage of energy remaining in the system is much higher for the lowest initial energy level considered, compared to the other energy levels. However, in general, consistent performance is observed, further demonstrating the robustness of the nonlinear NESbased design. An example of this is the energy remaining after one second when the NESs are unlocked; at the lowest energy level considered there is $58 \%$ of this energy remaining, while at the other (higher) initial energy levels, the energy remaining after one second is between $43 \%$ and 34\%. Furthermore, in all of the results presented, except for the lowest initial energy level, the energy remaining after 4 seconds is less than $2 \%$ with the NESs unlocked, while the energy remaining after 4 seconds with the NESs locked is $42 \%$. This further underscores the passive and adaptive capacity of the system of NESs to rapidly absorb and locally dissipate, and to scatter energy from low to higher frequencies at a fast time scale, over a broad range of impulse intensities.

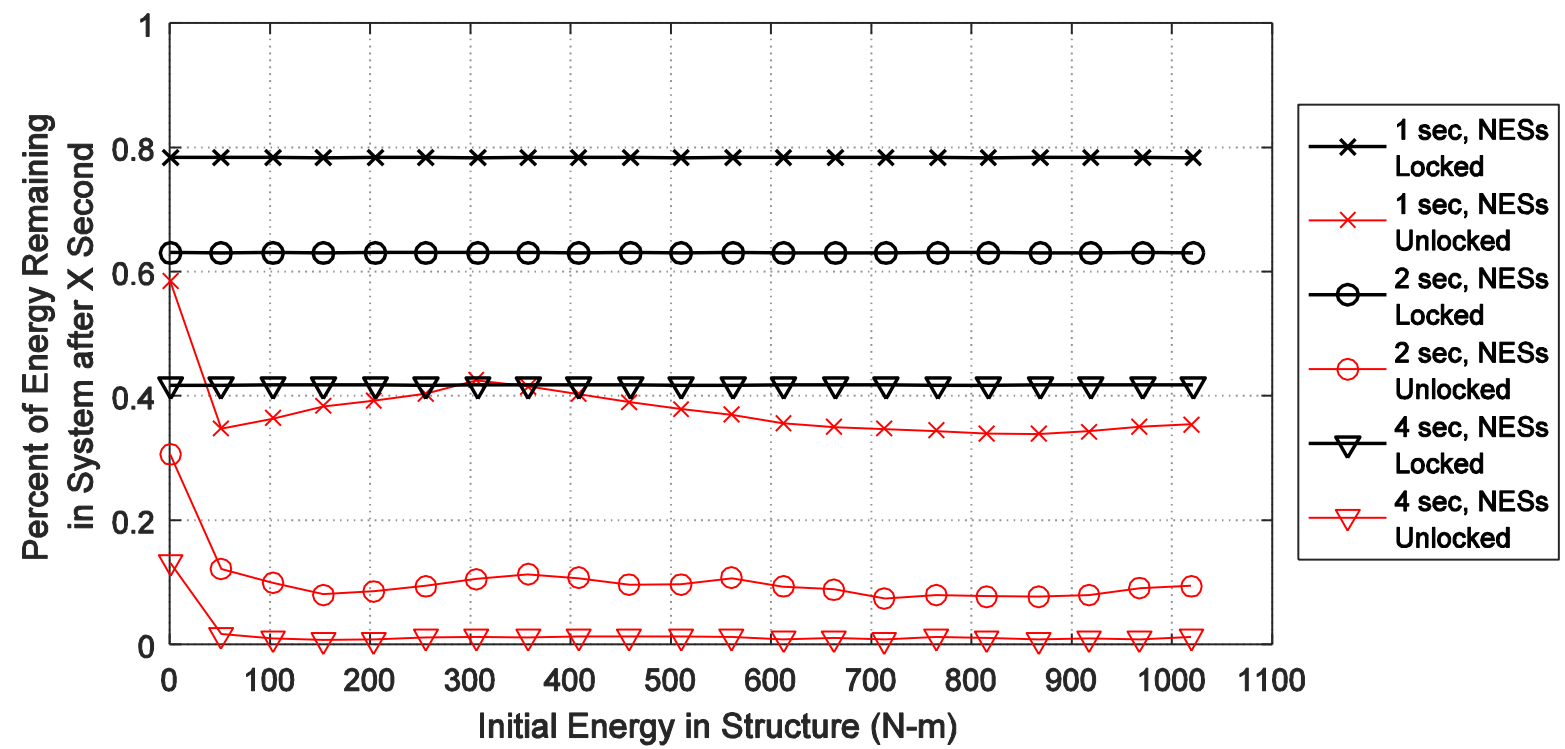

Figure 19. Percentage of energy remaining in structure after 1, 2, and 4 seconds over a range of initial energy levels. 
For many buildings, the first story is the critical portion of the structure; as a consequence of this, the reduction of the first-story drift is particularly important for a structural control system. Figure 20 depicts the ratio of the peak first-story drift when the NESs are unlocked to the corresponding peak when the NESs are locked over the range of initial energy levels consider in this analysis. This result shows that at most of the energy levels considered, a consistent reduction in peak story drift of about $15 \%$ was observed. Similar to the trends observed in Figure 19, the performance of the system of NESs was worst at the lowest energy level where an increase in drift of $11 \%$ was observed. While an increase in first story drift due to the system of NESs is not ideal, because this occurred at the lowest energy level, the magnitude of the drift is still relatively small.

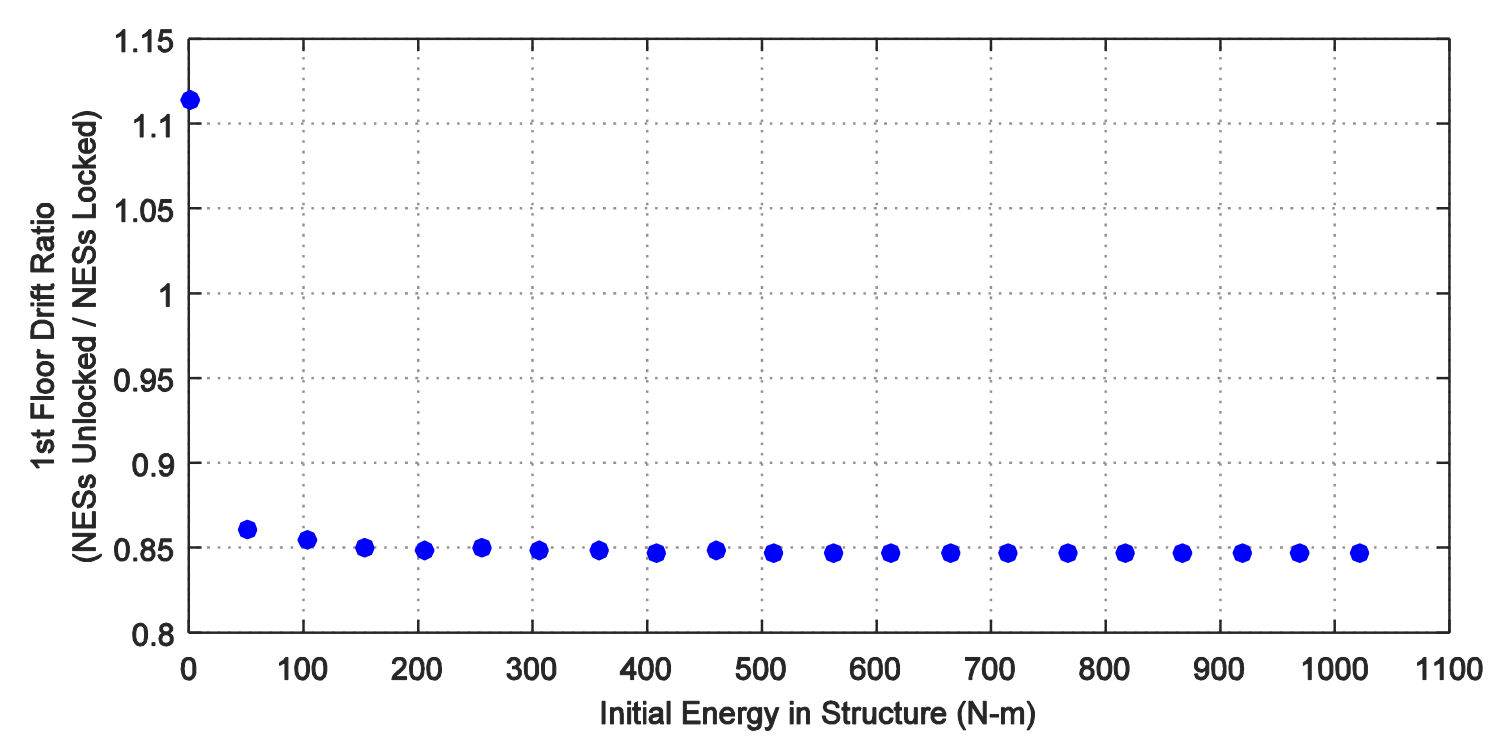

812 Figure 20. First story drift ratio comparing the NESs unlocked versus NESs locked cases over the range 813 of initial energy levels considered in this study.

\section{Conclusions}

In this work, the experimental blast testing of a large scale 9-story steel structure equipped with a system of nonlinear energy sinks (NESs) was examined. This represents the first experimental investigation of blast response attenuation with NESs. The system of NESs considered in this work included two different types of NESs. One type utilizes a smooth restoring force that is approximately cubic and is realized with specially shaped elastomeric bumpers, while the other incorporates a very weak linear restoring force and a mass undergoing one-sided vibro-impacts. The target parameters for this system of NESs were produced using a multiple-stage optimization technique and the results were used to guide the design of the physical system of NESs. The results of our testing program show that the system of NESs is capable of quickly diminishing the global response of the structure. 
The rapid reduction of the structural response is associated with broadband motion of the NESs, and significant transfer of energy from the lower modes of the structure to its higher modes. This nonlinear scattering of the blast energy in the frequency domain is very beneficial to the blast mitigation objective. First, by transferring energy in a fast scale from low to high frequencies the amplitude of the response rapidly decreases, and second, the higher modes have the inherent capacity to dissipate energy more effectively compared to the lower modes. Hence, the rapid nonlinear action of the Type VI NESs leads to a redistribution of blast energy in the modal space of the base structure and better "utilization" of its inherent damping capacity. We note at this point the capacity of the vibro-impact NESs for early action (i.e., they have a fast time scale), which enables them to affect the structural response during the highly energetic phase from the first cycle of the response. To the authors' knowledge such rapid structural response reduction by purely passive means is reported for the first time. Moreover, we note that through the vibro-impacts the Type VI NESs "feed" a portion of the blast energy to the Type I (smooth) NESs which are activated at a later phase of the motion; the ensuing local energy dissipation at their locations further enhances the dissipative capacity of the integrated base structure/NESs system.

Multiple tests with varying blast intensities demonstrated that, because of the strongly nonlinear and non-smooth nature of the system of NESs, this system can be effective across a wide range of load levels. Computational simulations, which utilized a model validated with the experimental data, also demonstrated that the system of NESs delivered consistent performance with a rapid reduction of energy in the system once a minimum load level had been exceeded. Additionally, the results of the experimental tests and the computational simulations demonstrated that the passive system of NESs studied in this work is capable of reducing peak demand on the structure due to this impulsive loading, as measured by the drift in the first-story and the strain in the first-story columns. This is one more benefit that our passive nonlinear design provides in meeting the blast mitigation objective.

\section{Acknowledgments}

This research program was sponsored by the Defense Advanced Research Projects Agency through grant HR0011-10-1-0077; Dr. Aaron Lazarus was the program manager. The content of this paper does not necessarily reflect the position or the policy of the Government, and no official endorsement should be inferred. The authors would like to acknowledge the support of US Army Corps of Engineers personnel, in particular Matthew Holmer, during the experimental portions of this research.

\section{References}

[1] C. L. Elliott, G. C. Mays, P. D. Smith, The protection of buildings against terrorism and disorder, Proc. ICE - Struct. Build. 94 (1992) 287-297. 
[2] T. Ngo, P. Mendis, A. Gupta, J. Ramsay, Blast loading and blast effects on structures-An overview, Electron. J. Struct. Eng. 7 (2007) 76-91.

[3] J. Luo, N.E. Wierschem, S.A. Hubbard, L.A. Fahnestock, D. Dane Quinn, D. Michael McFarland, B.F. Spencer, A.F. Vakakis, L.A. Bergman, Large-scale experimental evaluation and numerical simulation of a system of nonlinear energy sinks for seismic mitigation, Eng. Struct. 77 (2014) 34-48. doi:10.1016/j.engstruct.2014.07.020.

[4] D.D. Quinn, A.L. Triplett, A.F. Vakakis, L.A. Bergman, Energy Harvesting From Impulsive Loads Using Intentional Essential Nonlinearities, J. Vib. Acoust. 133 (2011) 011004.

[5] T.P. Sapsis, D.D. Quinn, A.F. Vakakis, L.A. Bergman, Effective Stiffening and Damping Enhancement of Structures With Strongly Nonlinear Local Attachments, J. Vib. Acoust. 134 (2012) 011016-12. doi:10.1115/1.4005005.

[6] F. Schmidt, C.H. Lamarque, Energy pumping for mechanical systems involving nonsmooth Saint-Venant terms, Int. J. Non-Linear Mech. 45 (2010) 866-875.

[7] M.A. Al-Shudeifat, N.E. Wierschem, D.D. Quinn, A.F. Vakakis, L.A. Bergman, B.F. Spencer, Numerical and Experimental Investigation of a Highly Effective Single-Sided Vibro-Impact Nonlinear Energy Sink for Shock Mitigation, Int. J. Non-Linear Mech. 52 (2013) 96-109. doi:10.1016/j.ijnonlinmec.2013.02.004.

[8] B. Vaurigaud, A.T. Savadkoohi, C.-H. Lamarque, Efficient Targeted Energy Transfer With Parallel Nonlinear Energy Sinks: Theory and Experiments, J. Comput. Nonlinear Dyn. 6 (2011) 041005. doi:10.1115/1.4003687.

[9] J. Wang, N.E. Wierschem, B.F. Spencer Jr, X. Lu, Track Nonlinear Energy Sink for Rapid Response Reduction in Building Structures, J. Eng. Mech. (2014). http://ascelibrary.org/doi/abs/10.1061/(ASCE)EM.1943-7889.0000824 (accessed August 19, 2014).

[10] N.E. Wierschem, D.D. Quinn, S.A. Hubbard, M.A. Al-Shudeifat, D.M. McFarland, J. Luo, L.A. Fahnestock, B.F. Spencer, A.F. Vakakis, L.A. Bergman, Passive Damping Enhancement of a Two-degree-of-freedom System Through a Strongly Nonlinear Twodegree-of-freedom Attachment, J. Sound Vib. $331 \quad$ (2012) 5393-5407. doi:10.1016/j.jsv.2012.06.023.

[11] N.E. Wierschem, J. Luo, M.A. Al-Shudeifat, S.A. Hubbard, R.J. Ott, L.A. Fahnestock, D.D. Quinn, D.M. McFarland, B.F. Spencer, A.F. Vakakis, L.A. Bergman, Experimental Testing and Numerical Simulation of a Six-Story Structure incorporating a Two Degree of Freedom Nonlinear Energy Sink, ASCE J. Struct. Eng. 140 (2014). doi:10.1061/(ASCE)ST.1943541X.0000978.

[12] V.I. Arnold, V.V. Kozlov, A.I. Neishtadt, Mathematical aspects of classical and celestial mechanics, 3rd ed., Springer, Berlin ; New York, 2006.

[13] G. Kerschen, A.F. Vakakis, Y.S. Lee, D.M. McFarland, L.A. Bergman, Toward a Fundamental Understanding of the Hilbert-Huang Transform in Nonlinear Structural Dynamics, J. Vib. Control. 14 (2008) 77 -105. doi:10.1177/1077546307079381.

[14] G. Kerschen, Y.S. Lee, A.F. Vakakis, D.M. McFarland, L.A. Bergman, Irreversible Passive Energy Transfer in Coupled Oscillators with Essential Nonlinearity, SIAM J. Appl. Math. 66 (2005) 648. doi:10.1137/040613706.

[15] Y.S. Lee, A.F. Vakakis, L.A. Bergman, D.M. McFarland, G. Kerschen, F. Nucera, S. Tsakirtzis, P.N. Panagopoulos, Passive non-linear targeted energy transfer and its 
applications to vibration absorption: a review, Proc. Inst. Mech. Eng. Part K J. Multi-Body Dyn. 222 (2008) 77-134. doi:10.1243/14644193JMBD118.

[16] Y.S. Lee, F. Nucera, A.F. Vakakis, D.M. McFarland, L.A. Bergman, Complex Structure of Periodic Orbits in an Oscillator with a Vibro-Impact Attachment, Vibro-Impact Dyn. Ocean Syst. Relat. Probl. (2009) 173-178.

[17] J. Luo, N.E. Wierschem, L.A. Fahnestock, B.F. Spencer, D.D. Quinn, D.M. McFarland, A.F. Vakakis, L.A. Bergman, Design, simulation and large-scale testing of an innovative vibration mitigation device employing essentially nonlinear elastomeric springs, Earthq. Eng. Struct. Dyn. 43 (2014) 1829-1851. doi:10.1002/eqe.2424.

[18] N.E. Wierschem et al., Structural Logic: Tailoring Stiffness and Damping of Large Scale Structures Via Passive Nonlinear Targeted Energy Transfer, DARPA Strategic Technology Office (STO), 2013.

[19] U.S. Army Engineer Research and Development Center, Triaxial Earthquake and Shock Simulator (TESS), (2008). http://134.164.46.151/pls/erdcpub/docs/erdc/docs/ERDCFactSheet_Facility_TESS.pdf (accessed January 8, 2013).

[20] J.R. Dormand, P.J. Prince, A family of embedded Runge-Kutta formulae, J. Comput. Appl. Math. 6 (1980) 19-26. doi:10.1016/0771-050X(80)90013-3.

[21] Matlab 8, The Mathworks, Natick, Massachusetts, 2012.

[22] D.D. Quinn, S.A. Hubbard, N.E. Wierschem, M.A. Al-Shudeifat, R.J. Ott, J. Luo, B.F. Spencer, D.M. McFarland, A.F. Vakakis, L.A. Bergman, Equivalent modal damping, stiffening and energy exchanges in multi-degree-of-freedom systems with strongly nonlinear attachments, Proc. Inst. Mech. Eng. Part K J. Multi-Body Dyn. 226 (2012) 122146. doi:10.1177/1464419311432671.

[23] J.R. Britt, D.E. Ranta, C.E. Joachim, BlastX code, version 4.2, user's manual, U.S. Army Engineering Research and Development Center, Vicksburg, MS, 2001.

[24] Department of Homeland Security Science and Technology Directorate, Reference Manual to Mitigate Potential Terrorist Attacks Against Buildings (FEMA-426/BIPS-06), 2nd ed., 2011.

[25] D. Labat, Recent advances in wavelet analyses: Part 1. A review of concepts, J. Hydrol. 314 (2005) 275-288.

[26] R.E. Kalman, A new approach to linear filtering and prediction problems, Trans. ASME J. Basic Eng. (1960) 35-45.

[27] R.E. Kalman, R.S. Bucy, New results in linear filtering and prediction theory, J. Fluids Eng. 83 (1961) 95-108. 